\title{
Shallow groundwater recharge mechanism and apparent age in the Ndop plain, northwest Cameroon
}

\author{
Mengnjo Jude Wirmvem • Mumbfu Ernestine Mimba • Brice Tchakam Kamtchueng • \\ Engome Regina Wotany • Tasin Godlove Bafon - Asobo Nkengmatia Elvis Asaah • \\ Wilson Yetoh Fantong $\cdot$ Samuel Ndonwi Ayonghe $\cdot$ Takeshi Ohba
}

Received: 1 October 2014/ Accepted: 5 February 2015/Published online: 20 February 2015

(C) The Author(s) 2015. This article is published with open access at Springerlink.com

\begin{abstract}
Knowledge of groundwater recharge and apparent age constitutes a valuable tool for its sustainable management. Accordingly, shallow groundwater $(n=72)$ in the Ndop plain has been investigated using the stable isotopes of oxygen $\left({ }^{18} \mathrm{O}\right)$ and hydrogen $\left({ }^{2} \mathrm{H}\right.$ or $\left.\mathrm{D}\right)$ and tritium $\left({ }^{3} \mathrm{H}\right)$ to determine the recharge process, timing and rate of recharge, and residence time. The shallow groundwater showed low variability in $\delta^{18} \mathrm{O}$ values $(-2.7$ to $4.1 \%$ ) and ${ }^{3} \mathrm{H}$ content (2.4-3.1 TU). The low variability suggests a similar origin, homogenous aquifer, good water mixing and storage capacity of the groundwater reservoir. Like surface water, a cluster of groundwater along the Ndop Meteoric Water Line (NMWL) and Global Meteoric Water Line indicates meteoric origin/recharge. The rainfall
\end{abstract}

\author{
M. J. Wirmvem (₫) · T. Ohba \\ Department of Chemistry, School of Science, Tokai University, \\ 4-1-1 Kitakaname, Hiratsuka, Kanagawa 259-1211, Japan \\ e-mail: judevom@yahoo.co.uk \\ M. E. Mimba · W. Y. Fantong \\ Institute of Mining and Geological Research, P.O. Box 4110, \\ Yaounde, Cameroon

\section{B. T. Kamtchueng} \\ Graduate School of Science and Engineering for Education, \\ University of Toyama, Gofuku 3190, Toyama 930-8555, Japan \\ E. R. Wotany · S. N. Ayonghe \\ Department of Environmental Science, Faculty Science, \\ University of Buea, Box 63, Buea, Cameroon \\ T. G. Bafon \\ Compagnie Miniere Du Cameroun, P.O Box 35561, Yaounde, \\ Cameroon
}

A. N. E. Asaah

Department of Earth and Planetary Sciences, Tokyo Institute of Technology, Tokyo 152-8551, Japan recharge occurs under low relative humidity conditions and negligible evaporation effect. About $80 \%$ of the recharge is from direct heterogeneous/diffuse local precipitation at low altitude $(<1,260 \mathrm{~m})$ within the Ndop plain. Approximately $20 \%$ is from high altitude precipitation (localised recharge) or is recharged by the numerous inflowing streams and rivers from high elevations. A homogenous cluster of $\delta$-values in groundwater (and surface water) between May and June monsoon rains on the NMWL suggests dominant recharge during these months. The recharge represents at least $16 \%$ (>251 $\mathrm{mm})$ of the annual rainfall $(1,540 \mathrm{~mm})$ indicating high annual recharge; high enough for development of the groundwater resource for agriculture. The ${ }^{3} \mathrm{H}$ content $(>2.4 \mathrm{TU})$ in groundwater indicates post-1952 recharged water with an estimated residence time $<30$ years, suggesting short subsurface circulation, and subsequently a renewable aquifer.

Keywords Stable isotopes - Tritium groundwater dating Groundwater recharge $\cdot$ Residence time $\cdot$ Ndop plain .

Northwest Cameroon

\section{Introduction}

Groundwater is increasingly a vital resource to human populations around the world (Clark and Fritz 1997; Winter et al. 1998) especially in Africa where economic development and poverty reduction programmes drive the development of groundwater resources (Adelana and MacDonald 2008; MacDonald et al. 2012; Lapworth et al. 2013). Thus, hydrological investigations are essential for its sustainable management. The hydrological studies to assess groundwater resources and develop sustainable management strategies require a variety of scientific 
information (IAEA 2006). These include groundwater origin, recharge mechanism, residence time (Clark and Fritz 1997; Njitchoua et al. 1997; Kortatsi 2006; Goni 2006; Lapworth et al. 2013), rate of recharge (Coplen et al. 2000; IAEA 2006; Negrel et al. 2011; Takounjou et al. 2011) and timing of recharge (Mbonu and Travi 1994; Oga et al. 2008; Taylor and Howard 1996). Besides chemical tracers, such information has been better provided by the use of oxygen $\left({ }^{18} \mathrm{O}\right)$ and hydrogen (D) isotopes (Acheampong and Hess 2000; Deshpande et al. 2003; Goni 2006; Lapworth et al. 2013), which are integral parts of the water molecule (Craig 1961; Clark and Fritz 1997; Gat 2010). A comparison of the concentration of ${ }^{3} \mathrm{H}$ in groundwater with the historical records of high concentrations above natural levels since 1952 (due to nuclear weapons testing and nuclear reactors) has been used as a dating tool for groundwater (Solomon and Cook 2000; Plummer et al. 2003; Han et al. 2006).

Given the vulnerability of Sub-Saharan Africa to the changing climate (EACC 2010; Bonsor et al. 2011), changes in the intensity of precipitation may affect the timing and rate of groundwater recharge (Owor et al. 2009). Groundwater recharge studies in the semi-arid regions of Sub-Saharan Africa (Adanu 1991; Favreau et al. 2009; Fantong et al. 2010; Lutz et al. 2011) and Equatorial East Africa (Nkotagu 1996; Taylor and Howard 1996; Owor et al. 2009; Taylor et al. 2013a, b) have reported recharge by heaviest and isotopically depleted monsoon rains. However, in groundwater recharge studies in the equatorial West Africa, recharge is by abundant but relatively less depleted rainfall events (Mbonu and Travi 1994; Oga et al. 2008; Wirmvem et al. 2014a). Similarly, different recharge rates have been reported across SubSaharan Africa (Taylor and Howard 1996; and references therein; Fantong et al. 2010; Takounjou et al. 2011; Yidana and Chegbeleh 2013). While different hydrological conditions may explain the varied magnitudes of groundwater recharge estimates, the variety of the used methods also complicates comparisons. Multiple techniques (including stable isotope tracers) can provide a better insight of recharge rates (Taylor and Howard 1996).

Although ${ }^{3} \mathrm{H}$ levels in the atmosphere and precipitation have declined (because of radioactive decay and cessation of atmospheric testing) to the low natural concentrations, groundwater dating has recently been successfully carried out in Sub-Saharan Africa using ${ }^{3} \mathrm{H}$ (Fantong et al. 2010; Dassi 2011; Huneau et al. 2011; Ako et al. 2012). As reported, groundwater in semi-arid northern Africa (Huneau et al. 2011; Lapworth et al. 2013), low-latitude of West Africa (Loehnert 1988; Acheampong and Hess 2000; Oga et al. 2008; Onugba and Aboh 2009; Ako et al. 2012; Lapworth et al. 2013), including Cameroon (Njitchoua et al. 1997; Ketchemen-Tandia et al. 2007; Fantong et al.
2010; Ako et al. 2012; Kamtchueng et al. unpublished data) principally contains post-1952 meteoric recharge waters, suggesting a renewable resource. Despite the significance of such studies in groundwater management, they are limited in most communities in Cameroon where shallow groundwater is a primary source of household water supply.

The Ndop Plain (North West Cameroon) is a semi-urban community with an estimated population of over 200,000 inhabitants. Given its agricultural potentials, the area constitutes a backbone to the Cameroon economy (Ndzeidze 2008; Fonge et al. 2012). Surface water sources, which are contaminated by floods during the rainy season, run dry in the dry season. Subsequently, the daily demand for domestic water is met primarily by shallow dug wells. As reported, the major ion chemistry of the groundwater in the area is within the WHO guidelines (Wirmvem et al. 2013). The meteoric origin, recharge timing and resilience of the groundwater to short-term climatic changes have been described (Wirmvem et al. 2014a). However, there is a lack of additional hydrological information (based on dense sampling) including the recharge rate and residence time of the groundwater for its management.

Accordingly, this paper presents a multi-environmental tracer $\left({ }^{18} \mathrm{O}, \mathrm{D}\right.$ and $\left.{ }^{3} \mathrm{H}\right)$ characterisation of groundwater and surface water in the Ndop plain based on a detailed sampling campaign. This information, in addition to the existing chemical data, has been used to determine the (1) recharge process, (2) timing and recharge rate, and (3) residence time of the groundwater. Given the dense sampling, the results from this study have provided additional hydrological information to improve an understanding of the groundwater system; hence, contributing a tool for groundwater management in the area. A comparison with related studies in Sub-Saharan Africa will provide some insight on groundwater recharge across the region.

\section{The study area}

Location and climate

The Ndop plain (Fig. 1) is an inter-mountain basin (ca. $1,100 \mathrm{~km}^{2}$ ) in northwest Cameroon, located between latitudes $5^{\circ} 42^{\prime}$ and $6^{\circ} 10^{\prime} \mathrm{N}$ and longitudes $10^{\circ} 11^{\prime}$ and $10^{\circ} 40^{\prime} \mathrm{E}$. It is bounded by a chain of volcanoes namely Mt. Bamboutous (west), Mt. Sabga (northwest), Mt. Oku (north), the Wainamah peak (northeast) and the Mbam Massif (east). These volcanoes form part of a 1,600 km long chain of Cenozoic volcanic centres that cut diagonally across Cameroon in the SW-NE trend called the Cameroon Volcanic Line (Tanyileke et al. 1996). From the surrounding mountain chain, the relief drops steeply from an average 
Fig. 1 Map of the study area showing the drainage, relief, water sampling points and tritium values in selected 'boreholes'. The spatial plot of tritium shows an even distribution. Rivers and streams from the surrounding mountains discharge in a concentric fashion into the Bamendjin dam in the south-central portion

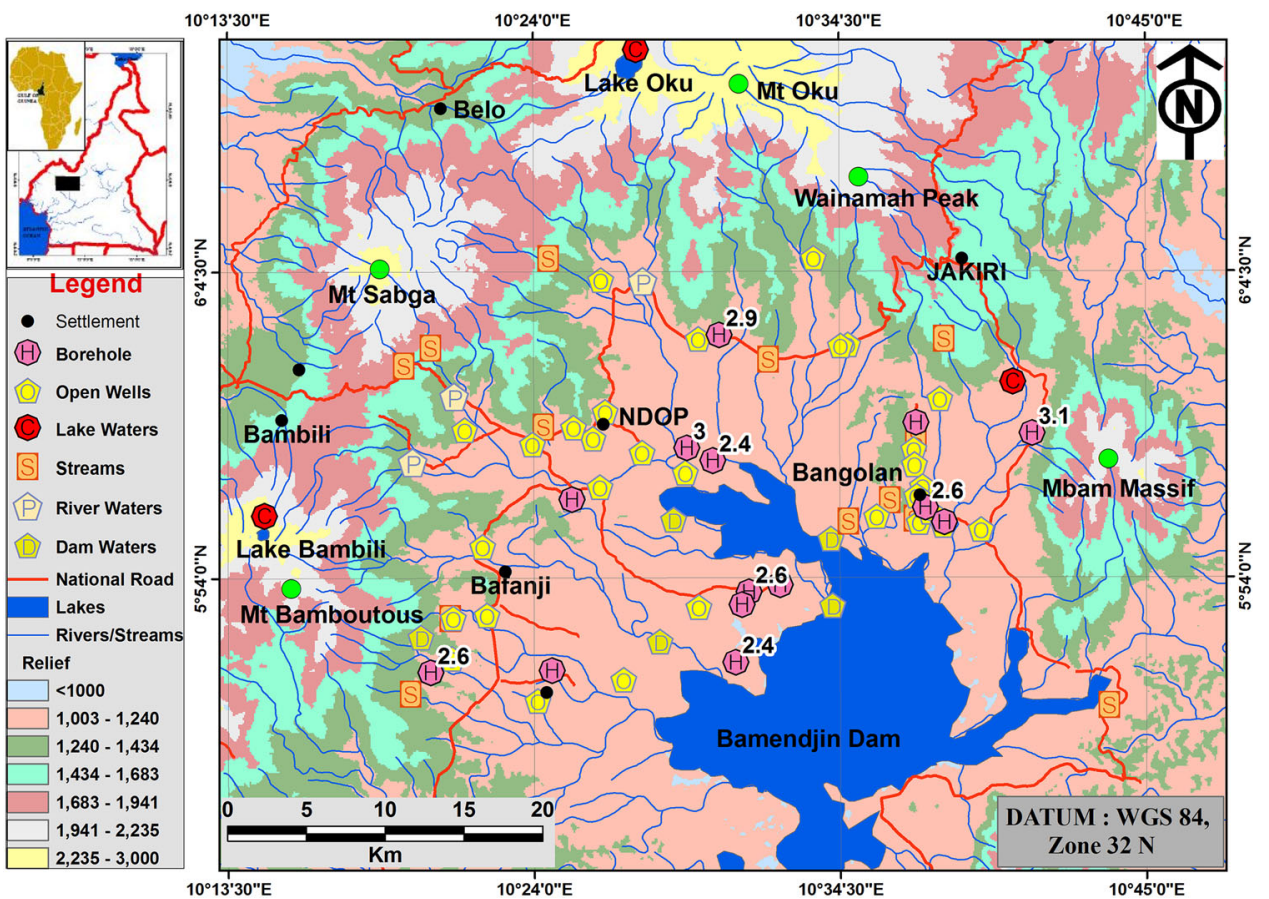

elevation of ca. 1,899-1,200 $\mathrm{m}$ at the margins of the plain, and then gently (though with some undulations) to $1,180 \mathrm{~m}$ above sea level (asl) in the south-central portion (Wirmvem et al. 2013).

The study area falls under the humid tropical equatorial climate type. Located only ca. $244 \mathrm{~km}$ northeast from the Atlantic Ocean (Fig. 1), it is subjected to the northward and southward movement of the inter-tropical convergence zone (ITCZ) and consequent effect of primary air masses (mainly the southwest monsoon). These, in addition to the high altitude of the surrounding mountainous relief, control the climate (Molua and Lambi 2006; Ndzeidze 2008). The ITCZ and southwest monsoon bring moisture-laden winds from the Gulf of Guinea, causing high relative humidity and rains (Neba 1999; Molua and Lambi 2006; Wirmvem et al. 2014b). It has two distinctive seasons, long rainy season (mid-March to mid-November) and short dry season (mid-November to mid-March). Annual rainfall ranges from 1,000 to 2,000 $\mathrm{mm}$ (Molua and Lambi 2006) with a mean value of $1,540 \mathrm{~mm}$, and an average temperature of $26{ }^{\circ} \mathrm{C}$ (Wirmvem et al. 2014b). A cooler climate prevails in adjacent high elevations, which record high rainfall than within the plain (Ndzeidze 2008).

\section{Geology and hydrogeology}

Geologically, the study area is a shallow Cenozoic 'sedimentary' basin underlain primarily by a consistent Precambrian granitic basement (Wirmvem et al. 2013). This basement constitutes part of a tectonically inactive African shield consisting mostly of Precambrian Basement
Complex rocks (Marzoli et al. 1999). The surrounding volcanoes are represented by voluminous Q-trachytes, and minor rhyolitic ignimbrites with slight to moderate alkaline basalts and minor basanite (Marzoli et al. 1999; Asaah et al. 2014).

Chemical weathering of the basement and surrounding volcanic rocks has produced thick unconsolidated sediments mainly of clay to sand sizes (Wirmvem et al. 2013). The major clay mineral is montmorillonite (smectite) with cristobalite, feldspars, ilmenite and heulandite as accessory minerals (Mache et al. 2013). The basement is largely covered with these sediments, but outcrops in certain portions. The weathered basement (regolith) and alkali-rich fluvial sediments constitute the shallow aquifer material in the plain. Percolation of water through the unconsolidated sediments forms the groundwater aquifer system with depths less than $30 \mathrm{~m}$ below the surface (Wirmvem et al. 2013, 2014a). Numerous rivers and streams discharge dendritically from the flanks of the mountainous chain facing the plain and recharge the Bamendjin dam (Fig. 1). The dam has modified the river/stream courses giving the area its wetland characteristics. During the rainy season, the area is usually flooded, particularly in the months of July, August and September (Ndzeidze 2008).

\section{Sampling and analytical methods}

A dense sampling campaign was undertaken at the peak of the dry season (January 2012) during which 72 water samples were collected from over 13 communities (Fig. 1). 
These included 32 open wells and 14 'boreholes' hereafter termed groundwater, 14 streams, 4 rivers, 5 dam waters, and 3 lakes (Lakes Bambili, Oku and Ber) hereafter called surface water. Surface water sources were collected under base flow conditions (no run-off influence) since the sampling was in the dry season. The locations and altitudes of the selected sampling points were identified in the field using a Garmin Vista CX GPS. New narrow mouth lowdensity polyethylene bottles $(100 \mathrm{ml})$ and Duran glass bottles $(500 \mathrm{ml})$ were used for stable isotopes and tritium $\left({ }^{3} \mathrm{H}\right)$ water sampling, respectively. The glass bottles contained screw caps bonded with polytetrafluoroethylenecoated silicone seal liners. In order to avoid contamination, the bottles were properly rinsed with distilled water and dried in the Laboratory. Further details on water sampling are described in Wirmvem (2014). Unfiltered samples from 8 selected 'boreholes' (representing the entire study area) were pumped into the glass bottles using a peristaltic pump. The samples were properly capped immediately after sampling and well-preserved in a cooler container containing ice blocks prior to air-flight to Japan for analyses.

Laboratory analysis of hydrogen and oxygen isotope ratios of the collected samples was carried out at Tokai University using a Cavity Ring-Down Spectrometer analyser (model L2120-i from PICARRO). The analysis followed the method described by Brand et al. (2009). The isotope ratio of $\mathrm{D} / \mathrm{H}$ and ${ }^{18} \mathrm{O} /{ }^{16} \mathrm{O}$ in the samples was expressed as per mille (\%o) deviation relative to Vienna-Standard Mean Ocean Water (V-SMOW) as follows:

$\delta(\%)=\left(R_{\text {sample }} / R_{\mathrm{V}-\mathrm{SMOW}}-1\right) \times 1000$

where $R$ is the ratio of the heavy to light isotopes $\left({ }^{18} \mathrm{O} /{ }^{16} \mathrm{O}\right.$ or $\mathrm{D} / \mathrm{H})$ in the sample or standard. The oxygen and hydrogen isotope ratios are hereinafter expressed as $\delta^{18} \mathrm{O}$ and $\delta \mathrm{D}$, respectively (or collectively as $\delta$-values). Total analytical precision for $\delta^{18} \mathrm{O}$ and $\delta \mathrm{D}$ was better than \pm 0.05 , $\pm 0.12 \%$, respectively. The stable isotopes $\left(\delta^{18} \mathrm{O}\right.$ and $\left.\delta \mathrm{D}\right)$ of monthly precipitation and weather records (Table 3 ) in the Ndop plain from Wirmvem et al. (2014b) were used in data interpretation.

Tritium counting was conducted (at Geo-Science Laboratory Co. Ltd, Nagoya, Japan) with a low-background liquid scintillation counter, Aloka model LB5 following electrolytic enrichment of ${ }^{3} \mathrm{H}$ by a factor of about 25 using $\mathrm{Fe}-\mathrm{Ni}$ electrodes. Total analytical precision was better than \pm 0.23 TU.

\section{Results}

From the measured water elevations and depth to wells (Table 1) referenced to sea level, a groundwater flow map in the Ndop has been produced (Fig. 2a). Higher altitude locations (e.g., Sabga) show high water elevation relative to the surrounding low altitude in the plain (Fig. 2a). This is due to groundwater discharge at high elevations through joints in the volcanic rocks from a likely perched aquifer system. As expected, a similar dendritic flow pattern to rivers can be seen in the groundwater flow map. However, at low-lying elevations, within the Ndop plain, a complex groundwater flow pattern is observed (Fig. 2a), suggesting groundwater mixing. A spatial view of $\delta^{18} \mathrm{O}$ shows various local flow systems (due to minor undulations) at lower elevations (Fig. 2b).

Tritium concentrations (Table 1), which showed an even spatial distribution, were low with a narrow range from 2.4 to 3.1 TU. Like ${ }^{3} \mathrm{H}$, the $\delta^{18} \mathrm{O}, \delta \mathrm{D}$ and $d$-excess of the analysed water (Table 1) showed a less compositional variability in both surface water and shallow groundwater (Table 2). The narrow ranges in ${ }^{3} \mathrm{H}$ and $\delta$-values further suggest a common origin, homogenous aquifer, water mixing (Fontes 1980) and good storage capacity of the groundwater reservoir. The $\delta^{18} \mathrm{O}$ and $\delta \mathrm{D}$ of the investigated water types were plotted on the conventional $\delta$-space diagram (Fig. 3). This was compared with the $\delta^{18} \mathrm{O}$ and $\delta \mathrm{D}$ of monthly precipitation (Table 3 ) and a corresponding Ndop Meteoric Water Line (NMWL) of Wirmvem et al. (2014b). Both surface water and groundwater showed significantly less variability in $\delta$-values (Fig. 3 ) than in monthly rainfall of the area (Table 3 ). The observed narrow cluster of all $\delta$ values in groundwater (between May and June rainfall) along the NMWL (Fig. 3) indicates meteoric water origin and recharge. The low mean $d$-excess of lake and dam waters (Table 2) and their plot to the upper right side of the NMWL with a slope of 3.7 (Fig. 3) suggest kinetic evaporation (Craig 1961).

Groundwater recharge elevation was determined from $\delta^{18} \mathrm{O}$ and altitude relationship (Coplen et al. 2000) of both surface water and groundwater. The water samples clustered into three groups, A, B and C (Fig. 4). About $92 \%$ of the samples, which included mainly open wells and 'boreholes', occurred at a low elevation ( $<1,260 \mathrm{~m}$ asl) in Group A (Fig. 4). These samples showed a less depleted $\delta^{18} \mathrm{O}$ band than the high altitude $(>1,449 \mathrm{~m}$ asl) Group B samples. Water recharged at high altitudes tends to have depleted $\delta^{18} \mathrm{O}$ (Gonfiantini et al. 1998; Gat 2010). Thus, the dominance of groundwater with less depleted $\delta^{18} \mathrm{O}$ at low elevations suggests low altitude recharge within the Ndop plain. Only a fraction of the group A samples, designated $\mathrm{A}^{1}$ had a $\delta^{18} \mathrm{O}$ band similar to relatively high altitude group B samples (Fig. 4) suggesting high altitude recharge or recharge by inflowing streams/rivers. Group $\mathrm{C}$ samples though at a higher elevation $(>2,243 \mathrm{~m}$ asl) had the most enriched $\delta^{18} \mathrm{O}$ band since it contained mostly 'stagnant' lake waters (subjected to the kinetic 
Table 1 Isotopic composition of groundwater (open wells, OW, 'boreholes', BH) and surface water (stream water, ST, river water, RW, dam water, DW, and lake water, LK) in the Ndop plain showing a general decrease in $\delta^{18} \mathrm{O}$ and $\delta \mathrm{D}$ from surface water to groundwater

\begin{tabular}{|c|c|c|c|c|c|c|c|c|c|c|c|c|}
\hline No. & Location & Latitude & Longitude & $\begin{array}{l}\text { Sample } \\
\text { No. }\end{array}$ & $\begin{array}{l}\text { Dtw }^{b} \\
(\mathrm{~m})\end{array}$ & $\begin{array}{l}\text { Alt. } \\
\text { (m) }\end{array}$ & $\begin{array}{l}\delta^{18} \mathrm{O} \\
(\%)\end{array}$ & $\begin{array}{l}\delta \mathrm{D} \\
(\%)\end{array}$ & $\begin{array}{l}d \text {-excess } \\
(\%)\end{array}$ & $\begin{array}{l}3 \mathrm{H} \\
(\mathrm{TU})\end{array}$ & $\begin{array}{l}\mathrm{TDS}^{\mathrm{b}} \\
(\mathrm{mg} / \mathrm{l})\end{array}$ & $\begin{array}{l}\mathrm{Cl}^{-\mathrm{b}} \\
(\mathrm{mg} / \mathrm{l})\end{array}$ \\
\hline 1 & Nkendipeh & $\mathrm{N}^{\prime} 06^{\circ} 02^{\prime} 11.9^{\prime \prime}$ & $\mathrm{E} 010^{\circ} 38^{\prime} 04.9^{\prime \prime}$ & OW06 & 15.5 & 1,218 & -3.2 & -16.9 & 8.3 & - & 9.8 & 0.1 \\
\hline 2 & Bangolan & $\mathrm{N}^{\prime} 06^{\circ} 05^{\prime} 12.3^{\prime \prime}$ & $\mathrm{E} 010^{\circ} 38^{\prime} 25.6^{\prime \prime}$ & OW07 & 7.3 & 1,188 & -3.2 & -17.4 & 8.5 & - & 18.2 & 0.2 \\
\hline 3 & Bangolan & $\mathrm{N} 0^{\circ} 02^{\prime} 49.7^{\prime \prime}$ & $\mathrm{E} 010^{\circ} 36^{\prime} 15.2^{\prime \prime}$ & OW09 & 12.3 & 1,183 & -3.6 & -18.0 & 10.7 & - & 16.3 & 0.1 \\
\hline 4 & Mbulung & $\mathrm{N}^{\circ} 6^{\circ} 06^{\prime} 28.86^{\prime \prime}$ & $\mathrm{E} 010^{\circ} 36^{\prime} 10.94^{\prime \prime}$ & OW10 & 4.3 & 1,184 & -3.1 & -15.4 & 9.3 & - & 12.4 & 0.1 \\
\hline 5 & Fongoh & $\mathrm{N}^{\circ} 6^{\circ} 04^{\prime} 57.69^{\prime \prime}$ & $\mathrm{E} 10^{\circ} 33^{\prime} 37.18^{\prime \prime}$ & OW18 & 9.7 & 1,176 & -3.5 & -18.4 & 9.5 & - & 5.9 & 0.1 \\
\hline 6 & Nkuwat & $\mathrm{N}^{\circ} 6^{\circ} 04^{\prime} 56.49^{\prime \prime}$ & $\mathrm{E} 010^{\circ} 33^{\prime} 30.42^{\prime \prime}$ & OW19 & 7.7 & 1,189 & -3.1 & -14.0 & 11.1 & - & 33.8 & 0.1 \\
\hline 7 & Mbuntoh & $\mathrm{N}^{\circ} 6^{\circ} 01^{\prime} 59.49^{\prime \prime}$ & $\mathrm{E} 010^{\circ} 34^{\prime} 47.53^{\prime \prime}$ & OW $20^{\mathrm{a}}$ & 11.2 & 1,180 & -3.8 & -18.1 & 12.3 & - & 38.4 & 1.1 \\
\hline 8 & Bangolan & $\mathrm{N}^{\circ} 6^{\circ} 01^{\prime} 29.77^{\prime \prime}$ & $\mathrm{E} 010^{\circ} 32^{\prime} 02.75^{\prime \prime}$ & OW21 & 1.7 & 1,163 & -2.8 & -11.2 & 10.8 & - & 36.4 & 2.1 \\
\hline 9 & Shupa & $\mathrm{N}^{\prime} 06^{\circ} 02^{\prime} 19.0^{\prime \prime}$ & $\mathrm{E} 010^{\circ} 30^{\prime} 23.3^{\prime \prime}$ & OW22 & 5.4 & 1,166 & -3.8 & -17.6 & 13.0 & - & 47.5 & 0.9 \\
\hline 10 & Massam & $\mathrm{N}^{\circ} 6^{\circ} 04^{\prime} 09.97^{\prime \prime}$ & $\mathrm{E} 010^{\circ} 27^{\prime} 44.91^{\prime \prime}$ & OW28 & 17.5 & 1,197 & -3.8 & -17.0 & 13.5 & - & 29.3 & 0.2 \\
\hline 11 & Poshi Wasi & $\mathrm{N}^{\circ} 6^{\circ} 12^{\prime} 07.59^{\prime \prime}$ & $\mathrm{E} 010^{\circ} 27^{\prime} 31.83^{\prime \prime}$ & OW32 & 6.3 & 1,192 & -3.7 & -19.1 & 10.5 & - & 28.6 & 0.4 \\
\hline 12 & Kwanso & $\mathrm{N}^{\prime} 6^{\circ} 12^{\prime} 25.7^{\prime \prime}$ & $\mathrm{E} 010^{\circ} 27^{\prime} 42.4^{\prime \prime}$ & OW37 & 5.5 & 1,260 & -3.6 & -18.4 & 10.5 & - & 42.3 & 0.1 \\
\hline 13 & Babessi & $\mathrm{N}^{\prime} 6^{\circ} 12^{\prime} 39.2^{\prime \prime}$ & $\mathrm{E} 010^{\circ} 27^{\prime} 39.5^{\prime \prime}$ & OW39 & 1.6 & 1,184 & -3.9 & -19.4 & 11.6 & - & 57.2 & 6.2 \\
\hline 14 & Banka & $\mathrm{N}^{\circ} 06^{\circ} 03^{\prime} 34.9^{\prime \prime}$ & $\mathrm{E} 010^{\circ} 25^{\prime} 10.2^{\prime \prime}$ & OW49 & 6.2 & 1,168 & -3.5 & -14.4 & 13.9 & - & 149.5 & 1.2 \\
\hline 15 & Messi & $\mathrm{N}^{\circ} 6^{\circ} 00^{\prime} 00.05^{\prime \prime}$ & $\mathrm{E} 010^{\circ} 26^{\prime} 17.8^{\prime \prime}$ & OW50 & 6.0 & 1,167 & -3.3 & -13.7 & 12.8 & - & 9.1 & 0.2 \\
\hline 16 & Mbanchoro & $\mathrm{N}^{\circ} 5^{\circ} 58^{\prime} 54.32^{\prime \prime}$ & $\mathrm{E} 010^{\circ} 26^{\prime} 41.16^{\prime \prime}$ & OW56 & 15.7 & 1,182 & -3.4 & -15.8 & 11.7 & - & 46.2 & 0.2 \\
\hline 17 & Mbehpo & $\mathrm{N}^{\circ} 6^{\circ} 58^{\prime} 17.06^{\prime \prime}$ & $\mathrm{E} 010^{\circ} 27^{\prime} 43.62^{\prime \prime}$ & OW59 & 3.9 & 1,188 & -3.1 & -11.9 & 13.3 & - & 29.3 & 0.2 \\
\hline 18 & Mbanti & $\mathrm{N}^{\circ} 5^{\circ} 57^{\prime} 36.01^{\prime \prime}$ & $\mathrm{E} 010^{\circ} 29^{\prime} 12.48^{\prime \prime}$ & OW62 & 2.2 & 1,189 & -3.3 & -14.2 & 12.0 & - & 16.9 & 0.5 \\
\hline 19 & Wundu & N05 $56^{\prime} 06.1^{\prime \prime}$ & $\mathrm{E} 010^{\circ} 28^{\prime} 52.3^{\prime \prime}$ & OW64 & 4.6 & 1,185 & -3.6 & -16.4 & 12.6 & - & 22.1 & 0.6 \\
\hline 20 & Gwanjif & $\mathrm{N}^{\circ} 5^{\circ} 53^{\prime} 30.86^{\prime \prime}$ & $\mathrm{E} 010^{\circ} 31^{\prime} 22.62^{\prime \prime}$ & OW66 & 2.2 & 1,209 & -3.9 & -17.5 & 13.9 & - & 10.4 & 0.2 \\
\hline 21 & Mbantap & $\mathrm{N}^{\circ} 5^{\circ} 53^{\prime} 45.57^{\prime \prime}$ & $\mathrm{E} 010^{\circ} 32^{\prime} 27.44^{\prime \prime}$ & OW67 & 4.7 & 1,204 & -3.7 & -17.0 & 12.8 & - & 24.7 & 0.5 \\
\hline 22 & Mbafang & $\mathrm{N}^{\circ} 5^{\circ} 53^{\prime} 02.33^{\prime \prime}$ & $\mathrm{E} 010^{\circ} 34^{\prime} 14.92^{\prime \prime}$ & OW72 & 10.2 & 1,179 & -3.3 & -15.2 & 10.9 & - & 10.4 & 0.2 \\
\hline 23 & Mundua & N05 $51^{\prime} 06.5^{\prime \prime}$ & E010 $30^{\prime} 55.4^{\prime \prime}$ & OW74 & 12.8 & 1,196 & -3.4 & -17.0 & 10.3 & - & 94.3 & 0.2 \\
\hline 24 & Kah-Finkwi & $\mathrm{N}^{\circ} 5^{\circ} 52^{\prime} 59.8^{\prime \prime}$ & $\mathrm{E} 010^{\circ} 29^{\prime} 39.4^{\prime \prime}$ & OW82 & 14.0 & 1,246 & -3.5 & -16.9 & 11.0 & - & 15.6 & 0.4 \\
\hline 25 & Mille & $\mathrm{N}^{\circ} 5^{\circ} 52^{\prime} 42.01^{\prime \prime}$ & $\mathrm{E} 010^{\circ} 29^{\prime} 12.87^{\prime \prime}$ & OW85 & 2.9 & 1,174 & -3.5 & -16.2 & 12.2 & - & 39.7 & 4.7 \\
\hline 26 & Ntenko & $\mathrm{N}^{\circ} 5^{\circ} 56^{\prime} 39.33^{\prime \prime}$ & $\mathrm{E} 010^{\circ} 26^{\prime} 50.51^{\prime \prime}$ & OW86 & 5.7 & 1,207 & -3.2 & -12.8 & 12.6 & - & 87.8 & 26.9 \\
\hline 27 & Mowefo & $\mathrm{N}^{\circ} 5^{\circ} 56^{\prime} 42.00^{\prime \prime}$ & $\mathrm{E} 010^{\circ} 25^{\prime} 19.07^{\prime \prime}$ & OW88 & 8.7 & 1,191 & -3.5 & -14.8 & 13.4 & - & 16.9 & 0.3 \\
\hline 28 & Bamessing & $\mathrm{N}^{\circ} 5^{\circ} 56^{\prime} 41.84^{\prime \prime}$ & $\mathrm{E} 010^{\circ} 25^{\prime} 18.89^{\prime \prime}$ & OW $90^{\mathrm{a}}$ & 5.3 & 1,205 & -3.5 & -15.2 & 13.1 & - & 102.7 & 3.1 \\
\hline 29 & Kabamo & N05 $55^{\prime} 04.7^{\prime \prime}$ & $\mathrm{E} 010^{\circ} 22^{\prime} 16.2^{\prime \prime}$ & OW $125^{\mathrm{a}}$ & 3.0 & 1,183 & -4.0 & -20.7 & 11.5 & - & 31.2 & 2.6 \\
\hline 30 & Meya & $\mathrm{N}^{\circ} 5^{\circ} 52^{\prime} 49.5^{\prime \prime}$ & $\mathrm{E} 010^{\circ} 23^{\prime} 42.7^{\prime \prime}$ & OW126 & 5.0 & 1,195 & -3.8 & -18.9 & 11.2 & - & 27.4 & 0.3 \\
\hline 31 & Munjong & $\mathrm{N}^{\circ} 5^{\circ} 52^{\prime} 43.2^{\prime \prime}$ & $\mathrm{E} 010^{\circ} 22^{\prime} 23.3^{\prime \prime}$ & OW $127^{\mathrm{a}}$ & 14.0 & 1,187 & -4.1 & -21.7 & 11.2 & - & 39.7 & 0.3 \\
\hline 32 & Bamali & N05 $52^{\prime} 37.88^{\prime \prime}$ & $\mathrm{E} 010^{\circ} 21^{\prime} 08.73^{\prime \prime}$ & OW129 & 7.0 & 1,175 & -3.9 & -18.5 & 12.8 & - & 81.9 & 6.6 \\
\hline 33 & Mambim & N05 $59^{\prime} 35.5^{\prime \prime}$ & $\mathrm{E} 010^{\circ} 37^{\prime} 10.3^{\prime \prime}$ & $\mathrm{BH} 02^{\mathrm{a}}$ & 8.5 & 1,194 & -3.8 & -19.9 & 10.2 & - & 26.0 & 0.1 \\
\hline 34 & Mbuntoh & N05 $59^{\prime} 18.6^{\prime \prime}$ & $\mathrm{E} 010^{\circ} 37^{\prime} 07.2^{\prime \prime}$ & BH11 & 7.0 & 1,177 & -3.4 & -16.5 & 10.8 & 2.6 & 28.0 & 0.2 \\
\hline 35 & Koutoupit & N05 $59^{\prime} 23.5^{\prime \prime}$ & $\mathrm{E} 010^{\circ} 37^{\prime} 07.3^{\prime \prime}$ & $\mathrm{BH} 29^{\mathrm{a}}$ & 6.6 & 1,182 & -3.8 & -18.0 & 12.6 & 3.1 & 85.2 & 1.0 \\
\hline 36 & Baba I & N05 $59^{\prime} 13.0^{\prime \prime}$ & $\mathrm{E} 010^{\circ} 36^{\prime} 41.7^{\prime \prime}$ & $\mathrm{BH} 41^{\mathrm{a}}$ & 10.0 & 1,194 & -3.7 & -17.3 & 11.9 & 2.9 & 176.8 & 3.0 \\
\hline 37 & Mbantoh & N05 $58^{\prime} 46.4^{\prime \prime}$ & $\mathrm{E} 010^{\circ} 37^{\prime} 07.1^{\prime \prime}$ & $\mathrm{BH} 52^{\mathrm{a}}$ & 11.0 & 1,170 & -3.6 & -15.6 & 13.1 & 2.6 & 29.3 & 0.2 \\
\hline 38 & Mbangkwo & N05 $58^{\prime} 23.4^{\prime \prime}$ & $\mathrm{E} 010^{\circ} 37^{\prime} 02.8^{\prime \prime}$ & BH53 & 11.2 & 1,173 & -3.6 & -17.4 & 11.6 & - & 22.1 & 0.7 \\
\hline 39 & Mbashe & N05 $57^{\prime} 52.3^{\prime \prime}$ & $\mathrm{E} 010^{\circ} 37^{\prime} 03.7^{\prime \prime}$ & $\mathrm{BH} 55^{\mathrm{a}}$ & 27.0 & 1,164 & -3.4 & -14.0 & 12.9 & 2.4 & 28.0 & 0.4 \\
\hline 40 & Mbehpo & N05 $57^{\prime} 39.5^{\prime \prime}$ & $\mathrm{E} 010^{\circ} 37^{\prime} 16.7^{\prime \prime}$ & $\mathrm{BH} 60^{\mathrm{a}}$ & 4.2 & 1,188 & -3.4 & -14.0 & 12.9 & - & 36.4 & 0.1 \\
\hline 41 & Balom & N05 $57^{\prime} 07.0^{\prime \prime}$ & $\mathrm{E} 010^{\circ} 37^{\prime} 20.5^{\prime \prime}$ & $\mathrm{BH} 70^{\mathrm{a}}$ & 14.2 & 1,215 & -3.7 & -15.9 & 13.5 & - & 33.8 & 0.2 \\
\hline 42 & Ekwo & N05 $56^{\prime} 00.48^{\prime \prime}$ & $\mathrm{E} 010^{\circ} 37^{\prime} 08.5^{\prime \prime}$ & BH73 & 11.7 & 1,173 & -3.7 & -17.3 & 12.3 & - & 183.3 & 2.7 \\
\hline 43 & Mbafo & N05 $56^{\prime} 24.2^{\prime \prime}$ & $\mathrm{E} 010^{\circ} 37^{\prime} 26.6^{\prime \prime}$ & BH76 & 14.5 & 1,177 & -3.6 & -17.0 & 11.4 & - & 55.9 & 0.3 \\
\hline 44 & Mesaw & N05 $56^{\prime} 17.6^{\prime \prime}$ & $\mathrm{E} 010^{\circ} 37^{\prime} 11.7^{\prime \prime}$ & BH77 & 14.4 & 1,179 & -3.3 & -13.1 & 13.3 & 2.4 & 21.5 & 0.6 \\
\hline 45 & Messi & N05 $56^{\prime} 0.02^{\prime \prime}$ & $\mathrm{E} 010^{\circ} 37^{\prime} 03.7^{\prime \prime}$ & $\mathrm{BH} 83^{\mathrm{a}}$ & 12.3 & 1,171 & -4.0 & -18.8 & 13.1 & 3.0 & 30.6 & 0.1 \\
\hline 46 & Bangolan & $\mathrm{N}^{\circ} 5^{\circ} 56^{\prime} 42.1^{\prime \prime}$ & $\mathrm{E} 010^{\circ} 36^{\prime} 29.0^{\prime \prime}$ & BH124 & 10.0 & 1,166 & -2.7 & -10.3 & 11.1 & - & 20.2 & 0.4 \\
\hline 47 & Mambim & $\mathrm{N}^{\prime} 6^{\circ} 01^{\prime} 16.8^{\prime \prime}$ & $\mathrm{E} 010^{\circ} 19^{\prime} 33.2^{\prime \prime}$ & ST05 & - & 1,194 & -3.1 & -16.9 & 8.0 & - & 44.9 & 0.1 \\
\hline
\end{tabular}


Table 1 continued

\begin{tabular}{|c|c|c|c|c|c|c|c|c|c|c|c|c|}
\hline No. & Location & Latitude & Longitude & $\begin{array}{l}\text { Sample } \\
\text { No. }\end{array}$ & $\begin{array}{l}\text { Dtw }^{b} \\
(m)\end{array}$ & $\begin{array}{l}\text { Alt. } \\
\text { (m) }\end{array}$ & $\begin{array}{l}\delta^{18} \mathrm{O} \\
(\% 0)\end{array}$ & $\begin{array}{l}\delta \mathrm{D} \\
(\% \mathrm{o})\end{array}$ & $\begin{array}{l}d \text {-excess } \\
(\% 0)\end{array}$ & $\begin{array}{l}3 \mathrm{H} \\
(\mathrm{TU})\end{array}$ & $\begin{array}{l}\mathrm{TDS}^{\mathrm{b}} \\
(\mathrm{mg} / \mathrm{l})\end{array}$ & $\begin{array}{l}\mathrm{Cl}^{-\mathrm{b}} \\
(\mathrm{mg} / \mathrm{l})\end{array}$ \\
\hline 48 & Ngohngoh & $\mathrm{N}^{\prime} 6^{\circ} 12^{\prime} 35.4^{\prime \prime}$ & $\mathrm{E} 010^{\circ} 41^{\prime} 15.2^{\prime \prime}$ & ST13 & - & 1,209 & -3.3 & -16.0 & 10.2 & - & 7.8 & 0.1 \\
\hline 49 & Fongoh & $\mathrm{N}^{\circ} 6^{\circ} 12^{\prime} 38.4^{\prime \prime}$ & $\mathrm{E} 010^{\circ} 41^{\prime} 38.0^{\prime \prime}$ & ST15 & - & 1,182 & -3.0 & -15.5 & 8.7 & - & 13.7 & 0.0 \\
\hline 50 & Masses & N06²0 49.5" & $\mathrm{E} 010^{\circ} 46^{\prime} 05.9^{\prime \prime}$ & ST17 & - & 1,155 & -3.1 & -15.6 & 9.7 & - & 19.5 & 0.1 \\
\hline 51 & Foumban & $\mathrm{N}^{\circ} 6^{\circ} 19^{\prime} 16.89^{\prime \prime}$ & $\mathrm{E} 010^{\circ} 48^{\prime} 24.26^{\prime \prime}$ & ST24 & - & 1,135 & -4.0 & -20.4 & 11.8 & - & 13.7 & 0.1 \\
\hline 52 & Bangambi & $\mathrm{N}^{\circ} 6^{\circ} 20^{\prime} 46.1^{\prime \prime}$ & $\mathrm{E} 010^{\circ} 46^{\prime} 16.8^{\prime \prime}$ & ST27 & - & 1,162 & -3.7 & -17.0 & 12.7 & - & 12.4 & 0.3 \\
\hline 53 & Mbonge & $\mathrm{N}^{\circ} 6^{\circ} 22^{\prime} 07.8^{\prime \prime}$ & $\mathrm{E} 010^{\circ} 47^{\prime} 14.1^{\prime \prime}$ & ST33 & - & 1,487 & -4.0 & -19.7 & 11.9 & - & 16.3 & 0.3 \\
\hline 54 & Ngingong & $\mathrm{N}^{\circ} 6^{\circ} 20^{\prime} 05.07^{\prime \prime}$ & $\mathrm{E} 10^{\circ} 45^{\prime} 47.55^{\prime \prime}$ & ST40 & - & 1,193 & -3.3 & -15.9 & 10.6 & - & 71.5 & 0.3 \\
\hline 55 & Gwanjif & $\mathrm{N}^{\circ} 6^{\circ} 18^{\prime} 37.9^{\prime \prime}$ & $\mathrm{E} 010^{\circ} 43^{\prime} 29.0^{\prime \prime}$ & ST65 & - & 1,205 & -3.3 & -13.7 & 12.5 & - & 38.4 & 1.0 \\
\hline 56 & Mulafi & $\mathrm{N}^{\prime} 6^{\circ} 10^{\prime} 52.3^{\prime \prime}$ & $\mathrm{E} 010^{\circ} 41^{\prime} 52.8^{\prime \prime}$ & ST68 & - & 1,261 & -3.5 & -14.4 & 13.2 & - & 28.6 & 0.2 \\
\hline 57 & Kah-Finkwi & $\mathrm{N}^{\circ} 6^{\circ} 00^{\prime} 36.5^{\prime \prime}$ & $\mathrm{E} 010^{\circ} 18^{\prime} 14.5^{\prime \prime}$ & ST80 & - & 1,449 & -4.0 & -18.8 & 13.5 & - & 113.8 & 0.1 \\
\hline 58 & Mofoh 1 & N05 $58^{\prime} 27.3^{\prime \prime}$ & $\mathrm{E} 010^{\circ} 10^{\prime} 50.1^{\prime \prime}$ & ST87 & - & 1,196 & -2.7 & -10.6 & 11.0 & - & 37.7 & 0.3 \\
\hline 59 & Sabga & $\mathrm{N}^{\circ} 5^{\circ} 57^{\prime} 17.2^{\prime \prime}$ & $\mathrm{E} 010^{\circ} 08^{\prime} 18.9^{\prime \prime}$ & ST104 & - & 1,529 & -3.9 & -18.8 & 12.6 & - & 29.9 & 0.1 \\
\hline 60 & Sabga & $\mathrm{N}^{\circ} 6^{\circ} 04^{\prime} 57.99^{\prime \prime}$ & $\mathrm{E} 010^{\circ} 26^{\prime} 27.38^{\prime \prime}$ & ST108 & - & 1,663 & -4.4 & -20.8 & 14.1 & - & 18.9 & 0.2 \\
\hline 61 & Ngoulam & N0550 $50^{\prime} 55.5^{\prime \prime}$ & $\mathrm{E} 010^{\circ} 20^{\prime} 17.6^{\prime \prime}$ & RW26 & - & 1,149 & -3.9 & -18.8 & 12.6 & - & 8.5 & 0.2 \\
\hline 62 & Babungo & N05 $50^{\prime} 45.7^{\prime \prime}$ & $\mathrm{E} 010^{\circ} 20^{\prime} 28.0^{\prime \prime}$ & RW42 & - & 1,176 & -4.1 & -19.6 & 13.0 & - & 52.0 & 0.4 \\
\hline 63 & Babanki & N05 $49^{\prime} 00.7^{\prime \prime}$ & $\mathrm{E} 010^{\circ} 25^{\prime} 07.3^{\prime \prime}$ & RW93 & - & 1,214 & -4.0 & -18.0 & 14.1 & - & 22.1 & 0.3 \\
\hline 64 & Ntukwe & $\mathrm{N}^{\circ} 5^{\circ} 49^{\prime} 47.3^{\prime \prime}$ & $\mathrm{E} 010^{\circ} 24^{\prime} 07.3^{\prime \prime}$ & RW94 & - & 1,215 & -3.1 & -13.1 & 11.7 & - & 25.4 & 0.2 \\
\hline 65 & Moshe & N05 58'56.52" & $\mathrm{E} 010^{\circ} 41^{\prime} 06.78^{\prime \prime}$ & DW16 & - & 1,153 & -1.7 & -9.6 & 4.1 & - & 20.8 & 0.4 \\
\hline 66 & Bamali & N06 01'15.9" & $\mathrm{E} 010^{\circ} 40^{\prime} 54.9^{\prime \prime}$ & DW51 & - & 1,155 & -2.4 & -9.3 & 9.7 & - & 21.5 & 0.6 \\
\hline 67 & Bampai & N06 00'07.5" & $\mathrm{E} 010^{\circ} 37^{\prime} 56.9^{\prime \prime}$ & DW54 & - & 1,154 & -2.9 & -11.6 & 11.9 & - & 28.6 & 1.1 \\
\hline 68 & Bambalang & N05 50'48.5" & $\mathrm{E} 010^{\circ} 42^{\prime} 28.2^{\prime \prime}$ & DW75 & - & 1,157 & -3.0 & -12.3 & 11.5 & - & 30.6 & 0.9 \\
\hline 69 & Mbanchoro & N05 56'06.1" & $\mathrm{E} 010^{\circ} 28^{\prime} 52.3^{\prime \prime}$ & DW128 & - & 1,164 & -2.6 & -11.9 & 8.8 & - & 29.9 & 1.9 \\
\hline 70 & Kibang-Ber & N06 $00^{\prime} 42.55^{\prime \prime}$ & $\mathrm{E} 010^{\circ} 40^{\prime} 26.89^{\prime \prime}$ & LK31 & - & 1,181 & -2.2 & -10.4 & 6.9 & - & 15.6 & 0.3 \\
\hline 71 & Lake Oku & N05 $51^{\prime} 15.4^{\prime \prime}$ & $\mathrm{E} 010^{\circ} 28^{\prime} 20.9^{\prime \prime}$ & LK43 & - & 2,243 & 0.0 & -1.7 & 2.0 & - & 29.3 & 0.5 \\
\hline 72 & Lake Bambili & N05 43'31.4" & $\mathrm{E} 010^{\circ} 52^{\prime} 04.9^{\prime \prime}$ & LK123 & - & 2,272 & -2.7 & -14.5 & 7.2 & - & 37.7 & 0.8 \\
\hline
\end{tabular}

Tritium shows a narrow range in groundwater

Average ${ }^{3} \mathrm{H}$ in groundwater is $2.7 \mathrm{TU}$

$d$-excess $=\delta \mathrm{D}-8 \times \delta^{18} \mathrm{O}$ (Dansgaard 1964)

$D t w$ depth to the water table (average: $8.75 \mathrm{~m}$ ), Alt. altitude above sea level

a Stable isotope data from Wirmvem et al. (2014a)

b Data from Wirmvem et al. (2013)

evaporation). Three selected streams, ST13, ST33 and ST108, unaffected by evaporation (high $d$-excess values) and located at different elevations gave a $\delta^{18} \mathrm{O}$ altitude gradient of $-0.24 \% / 100 \mathrm{~m}$. The gradient is within the range for the African continent (Clark and Fritz 1997).

\section{Discussion}

Recharge mechanism of groundwater

Given the low temperature $\left(<27{ }^{\circ} \mathrm{C}\right)$ of shallow groundwater in the study area (Wirmvem et al. 2013), the observed $\delta^{18} \mathrm{O}$ and $\delta \mathrm{D}$ values can be regarded as conservative in reactions with bedrock and soil materials
(Taylor and Howard 1996; Gat 2010; Kendall and Doctor 2011). Consequently, the $\delta$-values of the groundwater reflect the isotopic composition of recharging meteoric water. Two major processes that can affect the recharging rainfall are soil-zone processes and direct heterogeneous/diffuse or localised/focused rainfall infiltration (Taylor and Howard 1996). Besides the observed cluster of groundwater (and surface water) along the NMWL, the lack of correlation between $\delta^{18} \mathrm{O}$ versus TDS and $\mathrm{Cl}^{-}$(Fig. 5) further indicates that soil-zone evaporation prior to rainfall infiltration is not a significant process in the area. Given the relatively isolated nature of lake waters (Fig. 1), which further show enriched $\delta^{18} \mathrm{O}$ and $\delta \mathrm{D}$ values (Figs. 3, 4), localised recharge from the lakes is negligible. Only OW21 and BH124 water samples, located about 20 and $100 \mathrm{~m}$, 


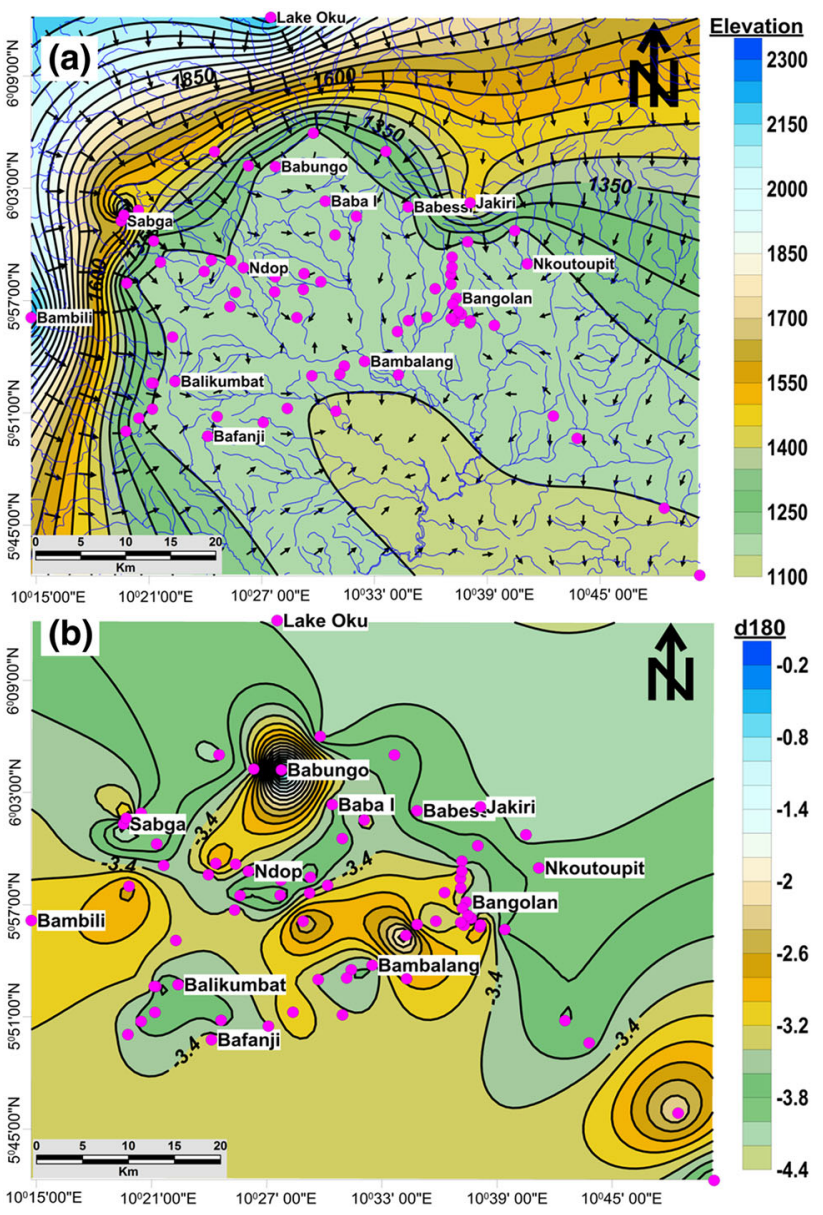

Fig. 2 Groundwater flow map in the Ndop plain (a) and a spatial view of $\delta^{18} \mathrm{O}$ in surface water and groundwater $(\mathbf{b})$. The pink dots represent sample points. Isotopically depleted water in $\delta^{18} \mathrm{O}$ is located at high elevations (e.g., Sabga) while relatively enriched water in $\delta^{18} \mathrm{O}$ occurs at low elevations

respectively, from the Bamendjin dam (southeast part of the study area), had exceptionally enriched $\delta$-values (Table 1). This suggests that recharge from the relatively enriched dam water may only be restricted to aquifer portions closer to the dam. Therefore, the most dominant groundwater recharge process is direct diffuse rainfall infiltration within the Ndop plain.

The high $d$-excess in groundwater (with $91 \%$ of the 46 groundwater samples having values above $10 \%$ ) also confirms direct rainfall infiltration (Kebede and Travi 2012) and recharge under low relative humidity conditions (Kendall and Doctor 2011). Similarity of $\delta^{18} \mathrm{O}, \delta \mathrm{D}$ and $d$-excess in streams and rivers to the groundwater indicates hydraulic connectivity with the shallow groundwater and potential lateral-vertical recharge or mixing within the plain.

From the aforementioned inferences, a conceptual model of groundwater recharge in the Ndop plain is proposed (Fig. 6). From the relationship in Fig. 4, $80 \%$ of the groundwater (open wells and 'boreholes') shows a less depleted $\delta^{18} \mathrm{O}$ band than the high elevation streams and rivers. This indicates that local precipitation at low altitude $(<1,260 \mathrm{~m})$ within the plain provides a high amount of recharge $(80 \%)$ to the shallow groundwater by a direct diffuse/heterogeneous mechanism. The recharging water rapidly infiltrates through numerous minor openings (enhanced by deforestation of land for agriculture) in the unconsolidated sediments under negligible evaporation effect. The water gradually percolates into the shallow groundwater aquifer, developing local flow systems (due to some undulations in the plain). High altitude localised recharge or recharge by inflowing streams and rivers only contributes $20 \%$ to groundwater recharge. Based on the shallow nature of the aquifer and narrow range in $\delta$-values and ${ }^{3} \mathrm{H}$, the groundwater aquifer is likely unconfined; hence associated with local flow systems within the plain as earlier observed. Such local flow systems are the most dynamic and have the greatest interchange with the surface (Winter et al. 1998); hence, the diffuse rainfall recharge within the Ndop plain.

Table 2 Statistical summary of stable isotope data of groundwater and surface water in the Ndop plain

\begin{tabular}{|c|c|c|c|c|c|c|c|c|c|c|}
\hline \multirow[t]{2}{*}{ Source } & \multicolumn{3}{|c|}{$\delta^{18} \mathrm{O}(\% 0)$} & \multicolumn{3}{|l|}{$\delta \mathrm{D}(\%)$} & \multicolumn{3}{|c|}{$d$-excess $(\% o)$} & \multirow{2}{*}{$\begin{array}{l}\text { Alt. (m) } \\
\text { Mean }\end{array}$} \\
\hline & Min. & Max. & Mean & Min. & Max. & Mean & Min. & Max. & Mean & \\
\hline $\mathrm{BH}(n=14)$ & -3.99 & -2.67 & -3.53 & -19.88 & -10.25 & -16.07 & 10.18 & 13.5 & 12.2 & 1,180 \\
\hline OW $(n=32)$ & -4.11 & -2.75 & -3.52 & -21.65 & -11.21 & -16.55 & 8.34 & 13.87 & 11.64 & 1,191 \\
\hline $\mathrm{GW}(n=46)$ & -4.11 & -2.67 & -3.53 & -21.65 & -10.25 & -16.4 & 8.34 & 13.87 & 11.81 & 1,188 \\
\hline $\mathrm{RW}(n=04)$ & -4.07 & -3.09 & -3.78 & -19.57 & -13.08 & -17.37 & 11.66 & 14.07 & 12.82 & 1,189 \\
\hline $\mathrm{ST}(n=14)$ & -4.35 & -2.71 & -3.52 & -20.78 & -10.62 & -16.72 & 7.99 & 14.06 & 11.46 & 1,287 \\
\hline $\mathrm{DW}(n=05)$ & -2.97 & -1.71 & -2.51 & -12.33 & -9.33 & -10.94 & 4.07 & 11.86 & 9.19 & 1,157 \\
\hline LK $(n=03)$ & -2.17 & -0.03 & -1.63 & -14.45 & -1.69 & -8.86 & 1.96 & 7.23 & 5.36 & 1,899 \\
\hline $\mathrm{SW}(n=26)$ & -4.34 & -0.03 & -3.15 & -20.78 & -1.69 & -14.8 & 1.96 & 14.07 & 10.53 & 1,317 \\
\hline All $(n=72)$ & -4.35 & -0.03 & -3.39 & -21.65 & -1.69 & -15.82 & 1.96 & 14.07 & 11.34 & 1,235 \\
\hline
\end{tabular}

$B H$ 'boreholes', $O W$ open well, $G W$ groundwater (BH and $\mathrm{OW}$ ), $R W$ river water, $S T$ stream water, $D W$ dam water, $L K$ lake water, $S W$ surface water (RW, ST, DW and LK), Alt. altitude above sea level 


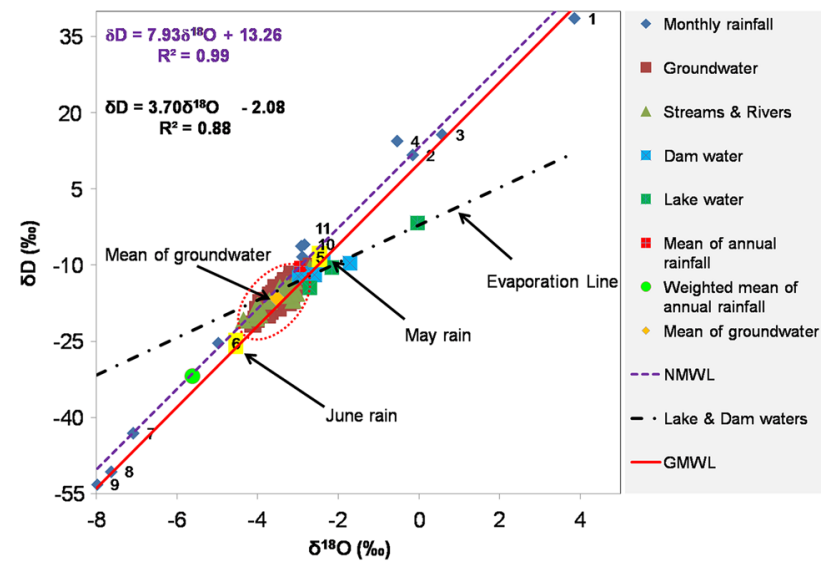

Fig. 3 A $\delta^{18} \mathrm{O}-\delta \mathrm{D}$ relationship of groundwater, surface water and rainfall in the Ndop plain. The numbers 1-11 represent monthly rainfall from January to November, respectively. There is a single cluster of water samples between May and June rains along the Ndop Meteoric Water Line (NMWL), and Global Meteoric Water Line (GMWL) of Craig (1961). Precipitation data are from Table 3

Timing and rate of groundwater recharge

A comparison of isotopic compositions between precipitation and groundwater can reveal the timing of groundwater recharge if precipitation shows distinctive seasonal variations in isotopic composition (Mbonu and Travi 1994; Nkotagu 1996; Taylor and Howard 1996; Deshpande et al. 2003; Ma et al. 2013) as in the study area (Table 3). As observed, the $\delta^{18} \mathrm{O}$ and $\delta \mathrm{D}$ (and average $\delta$ value) in groundwater narrowly clustered between those of May and June abundant monsoon rains. Despite the observed discharge of streams and rivers at higher altitudes, the range in their isotopic composition is also between these months (Fig. 3). The intersection of the evaporation line and the NMWL which usually represents the initial isotopic composition of inflow waters (Dincer 1968, Dassi 2011; Kebede and Travi 2012) into the lake and dam waters also lies between May and June rains. October-November rains show similar $\delta$-values to May precipitation (Fig. 3). The absence of enriched $\delta$-values of the low January-April pre-monsoon showers in groundwater rules out any significant recharge during these months. Interestingly, and as observed elsewhere in Equatorial West Africa, Nigeria (Mbonu and Travi 1994) and Ivory Coast (Oga et al. 2008), the $\delta^{18} \mathrm{O}$ and $\delta \mathrm{D}$ signatures of the most depleted and heaviest July-September monsoon precipitation (Table 3; Fig. 3) are clearly absent in the groundwater (Fig. 3). This indicates insignificant recharge during the heaviest rains (Table 3). Since the $\delta$ values of the groundwater, streams, and rivers are not significantly affected by evaporation, the dominant recharge period is likely from the abundant May to June monsoon rains. This have earlier reported in the area (Wirmvem et al. 2014a). A minor amount of recharge occurs during October-November post-monsoon showers.

During the light January-April pre-monsoon rainfall, the low relative humidity conditions prevail in the area (Table 3). The low humidity probably leads to high isotopic exchange and partial evaporation between the falling rain drops and the environmental vapour (Gat 2010); hence, the enriched $\delta$-values of rainfall (Table 3). The evaporation likely results to negligible groundwater recharge. By July, the vadose zone of the soil becomes saturated with the heavy May-June precipitation that directly percolates into the shallow unconfined aquifer. The heaviest July-September rains likely contribute to runoff

Table 3 Isotopic composition of monthly rainfall and weather records in the Ndop plain (2012) showing a progressive decrease in $\delta^{18} \mathrm{O}$ and $\delta \mathrm{D}$ with an increase in rainfall amounts (excerpt from Wirmvem et al. 2014b)

\begin{tabular}{|c|c|c|c|c|c|c|}
\hline Month & $\delta^{18} \mathrm{O}(\%)$ & $\delta \mathrm{D}(\%)$ & $d$-excess $(\% 0)$ & Rainfall (mm) & Relative humidity (\%) & $\overline{\text { Temperature }\left({ }^{\circ} \mathrm{C}\right)}$ \\
\hline January & 3.86 & 38.61 & 7.73 & 3 & 33 & 29.1 \\
\hline February & -0.15 & 11.67 & 12.87 & 63 & 36 & 24.3 \\
\hline March & 0.58 & 15.69 & 11.04 & 10 & 45 & 25.6 \\
\hline April & -0.54 & 14.44 & 18.74 & 89 & 50 & 24.9 \\
\hline May & -2.89 & -8.34 & 14.78 & 139 & 55 & 25.4 \\
\hline June & -4.97 & -25.38 & 14.39 & 112 & 54 & 24.5 \\
\hline July & -7.09 & -43.10 & 13.6 & 151 & 68 & 24.7 \\
\hline August & -7.64 & -50.66 & 10.42 & 278 & 79 & 24.2 \\
\hline September & -7.98 & -53.18 & 10.7 & 481 & 81 & 23.0 \\
\hline October & -2.84 & -5.88 & 16.87 & 133 & 50 & 25.9 \\
\hline November & -2.91 & -6.21 & 17.1 & 81 & 40 & 27.5 \\
\hline December & - & - & - & - & 38 & 28.8 \\
\hline Mean & -2.96 & -10.21 & 13.48 & 1,540 & 53.73 & 25.5 \\
\hline Volume weighted mean & -5.61 & -31.93 & 12.99 & - & - & - \\
\hline
\end{tabular}




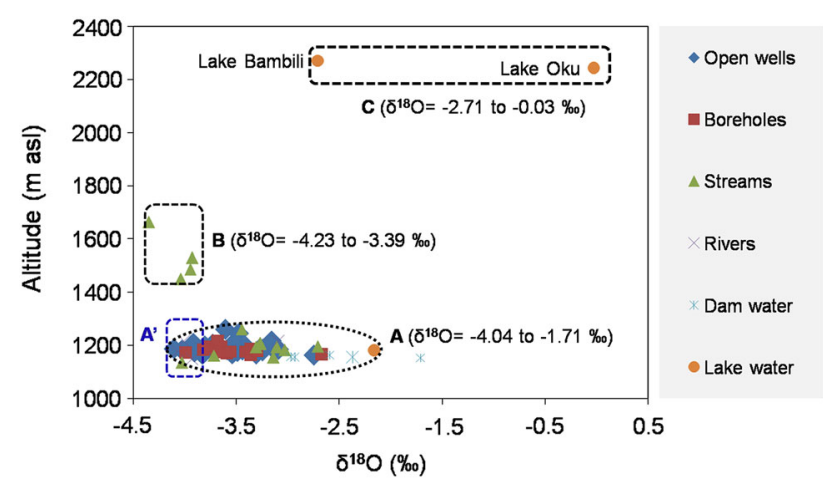

Fig. 4 Plot of $\delta^{18} \mathrm{O}$ in groundwater and surface water as a function of elevation in the study area. The water types cluster into 3 groups: $A(92 \%), B(5 \%)$ and $C(3 \%)$

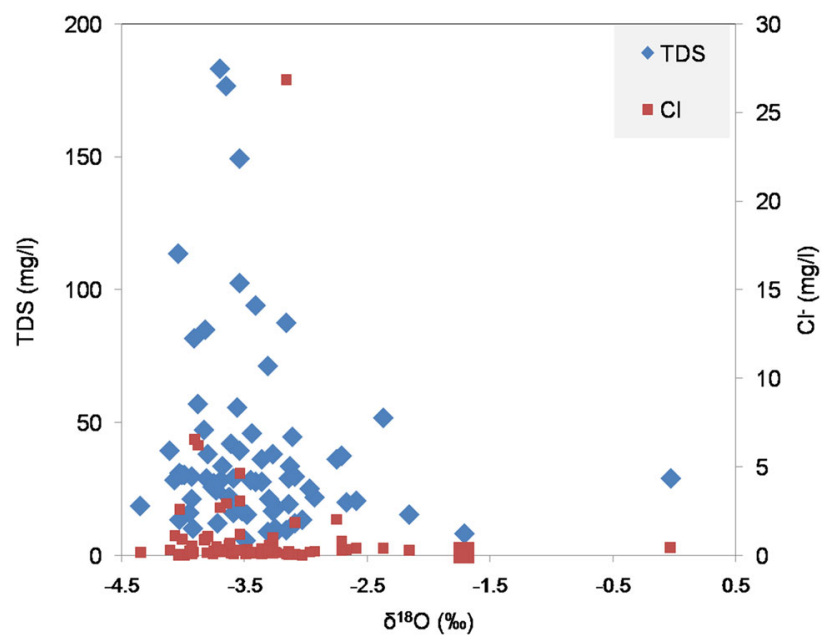

Fig. 5 Plot of $\delta^{18} \mathrm{O}$ in groundwater and surface water as a function of TDS and $\mathrm{Cl}^{-}$. There is an increase in TDS and $\mathrm{Cl}^{-}$without a corresponding increase in $\delta^{18} \mathrm{O}$ in both surface water and groundwater

and floods (Mbonu and Travi 1994; Oga et al. 2008) which are common during these months in the study area (Ndzeidze 2008). The period after which the vadose zone reaches its threshold capacity to hold water (July-September) is characterized by high relative humidity (Table 3). This results to minimal evaporation from the vadose zone such that groundwater recharge occurs under negligible evaporation effect. The runoff and floods from the July-September heaviest rains instead contribute significantly in recharging the Bamendjin dam (Fig. 1) as established by the annual recurrence increase in dam water from August (Personal communication with the Divisional Delegate of Agriculture and Rural Development, Ndop).

While the semi-arid regions of Sub-Saharan Africa (Adanu 1991; Favreau et al. 2009; Fantong et al. 2010; Lutz et al. 2011) and Equatorial regions of East Africa
(Nkotagu 1996; Taylor and Howard 1996; Owor et al. 2009; Taylor et al. 2013a) show recharge by the heaviest monsoon rains, only the abundant (and not the heaviest) monsoon rains recharge groundwater in Equatorial West Africa (Mbonu and Travi 1994; Oga et al. 2008; Wirmvem et al. 2014a). The disparity may be due to varied hydrological conditions (under a changing climate) including sources and atmospheric circulation of moisture (Taupin et al. 2000).

The observed similarity in $\delta^{18} \mathrm{O}$ values of the groundwater has also been reported in shallow groundwater (of recent meteoric origin) in some studies at the tropical low latitudes of West Africa (Table 4; Fig. 7). These regions, which show similar distinctive seasonal variations in rainfall, are located at low latitudes and $<244 \mathrm{~km}$ northward from the Gulf of Guinea (Fig. 7). Thus, they have a similar moisture source from the Atlantic Ocean (Taupin et al. 2000) as further confirmed by $d$-excess values close to $10 \%$, characteristic of low-latitude rains (Dansgaard 1964). The similar and narrow range in $\delta$-values of the groundwater in these studies indicates that modern rainfall recharge of groundwater in the tropical low latitudes of Equatorial West Africa occurs through a similar process and limited time of the year.

Based on the total rainfall during May and June $(251 \mathrm{~mm})$ relative to the annual precipitation $(1,540 \mathrm{~mm})$, and assuming negligible evaporation or runoff during these months, the estimated recharge rate is at least $16 \%$ $(>251 \mathrm{~mm})$ of the annual rainfall. The high recharge is probably enhanced by deforestation of the land for subsistence rainfed farming (Ibrahim et al. 2014) that constitutes over $60 \%$ of the land cover within the plain (Ndzeidze 2008). Similar high recharge rates have been reported in Uganda and Zambia (Taylor and Howard 1996 and references therein), and Ghana (Asomaning 1992; Yidana and Chegbeleh 2013) regardless of the varied methods used. Contrarily, relatively low recharge rates have been shown to occur in semi-arid regions of SubSaharan Africa (Fantong et al. 2010) and other Equatorial regions of Africa (Kenya, Uganda, Zimbabwe and Zambia) (Taylor and Howard 1996) including Yaounde, Cameroon (Takounjou et al. 2011). Such variations may be due to different hydrological conditions/or methods used (Taylor and Howard 1996).

Although the stable isotope data in the rain used in this study are not long-term data, it is assumed to represent recent past rainfall in the study area. This is supported by the fact that monthly rainfall patterns and amounts since 1960 to over 1990 in northwest Cameroon have not significantly changed (Neba 1999; Ayonghe 2001; Molua and Lambi 2006). 
Fig. 6 Conceptual model of groundwater recharge in the Ndop plain. The vadose zone (A) and saturated zone (B) comprise in situ weathered basement material and alkalirich fluvial sediments from the surrounding mountain chain. The saturated zone ranges from 4 to $30 \mathrm{~m}$ below the ground surface

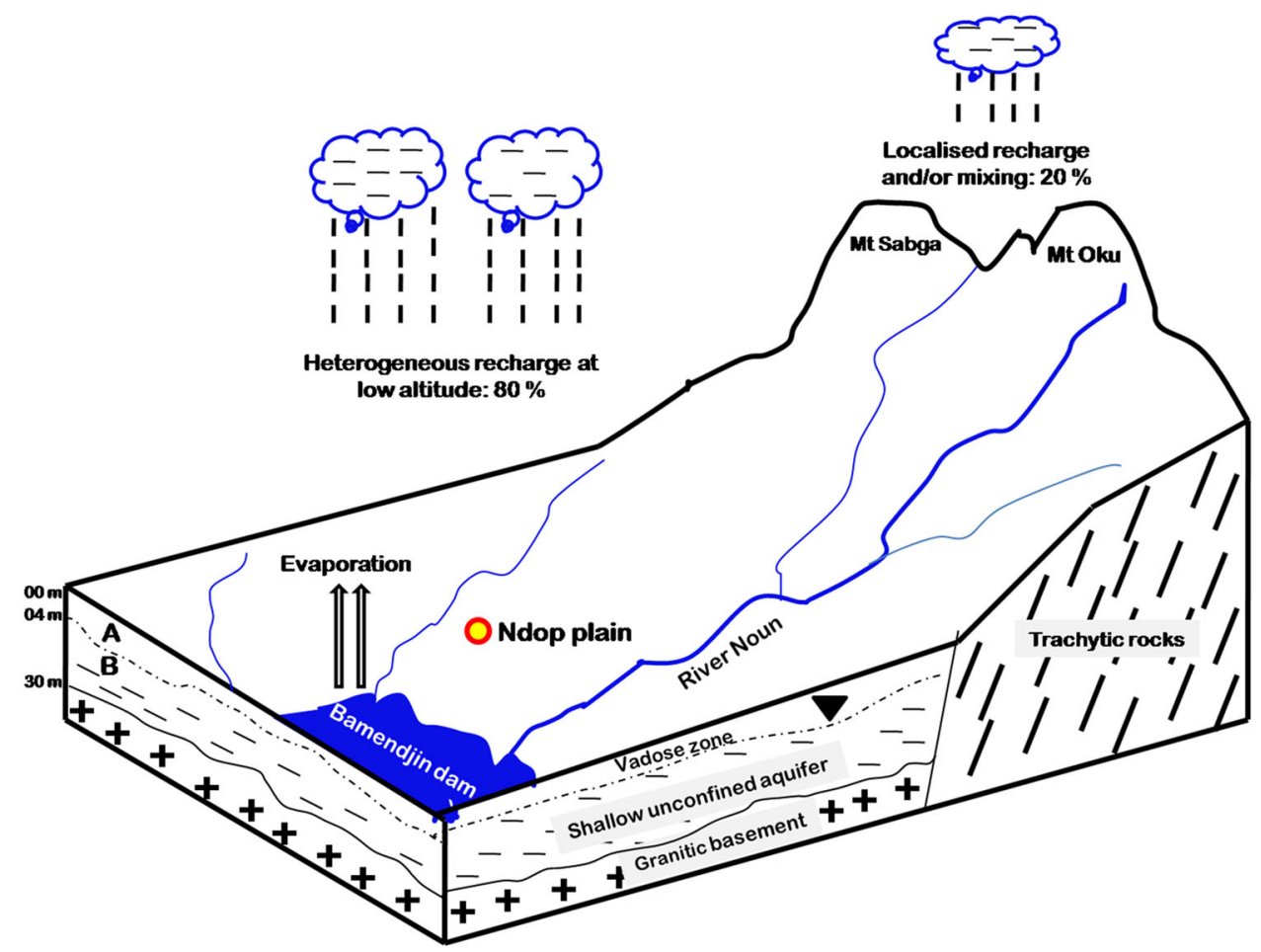

Table 4 The $\delta^{18} \mathrm{O}, \delta \mathrm{D}$ and $d$-excess in modern shallow groundwater from some studies in tropical low latitudes of western Africa compared to this study

\begin{tabular}{llllllllll}
\hline Location & Latitude & $\begin{array}{l}\text { Distance } \\
(\mathrm{km})\end{array}$ & $\begin{array}{l}\text { ca. Alt. } \\
(\mathrm{m})\end{array}$ & $\begin{array}{l}\text { Min. } \\
\delta^{18} \mathrm{O}\end{array}$ & $\begin{array}{l}\text { Max. } \\
\delta^{18} \mathrm{O}\end{array}$ & $\begin{array}{l}\text { Mean } \\
\delta^{18} \mathrm{O}\end{array}$ & $\begin{array}{l}\text { Mean } \\
\delta \mathrm{D}\end{array}$ & $d$-excess & Reference \\
\hline Ndop plain, CMR & $5^{\circ} 42^{\prime}-6^{\circ} 10^{\prime} \mathrm{N}$ & $<244$ & 1,200 & -4.1 & -2.7 & -3.5 & -16.4 & 11.8 & This study \\
Mbanga plains, CMR & $4^{\circ} 30^{\prime}-4^{\circ} 53^{\prime} \mathrm{N}$ & $<70$ & 500 & -4.2 & -2.1 & -3.2 & -15.3 & 10.3 & Ako et al. (2012) \\
Southern Ivory Coast & $5^{\circ} 00^{\prime}-5^{\circ} 30^{\prime} \mathrm{N}$ & $<4$ & 8 & -3.4 & -2.4 & -3.0 & - & - & Oga et al. (2008) \\
Accra plains, Ghana & $5^{\circ} 30^{\prime}-6^{\circ} 15^{\prime} \mathrm{N}$ & $<35$ & 70 & -3.9 & -2.1 & -3.0 & -14.1 & 10.0 & Kortatsi (2006) \\
SVSB, Ghana & $6^{\circ} 45^{\prime}-7^{\circ} 15^{\prime} \mathrm{N}$ & $<169$ & 100 & -4.2 & -2.6 & -3.0 & -14.0 & 10.0 & Acheampong and Hess (2000) \\
Southwest Nigeria & $6^{\circ} 22^{\prime}-7^{\circ} 18^{\prime} \mathrm{N}$ & $<120$ & 90 & -4.4 & -2.8 & -3.6 & -16.6 & 11.7 & Loehnert (1988) \\
Abeokuta, Nigeria & $6^{\circ} 41^{\prime}-7^{\circ} 15^{\prime} \mathrm{N}$ & $<100$ & 59 & -3.8 & -2.4 & -3.0 & -14.2 & 09.8 & Lapworth et al. (2013) \\
\hline
\end{tabular}

All latitudes except in Oga et al. (2008), Kortatsi (2006), Acheampong and Hess (2000) are located east of GMT

CMR Cameroon, SVSB southern Voltaian Sedimentary Basin, Distance is from the Atlantic coast, $c a$. Alt. approximate altitude (above sea level). Min minimum, Max maximum, $\delta$-values and $d$-excess are in \%o

Apparent recharge period: residence time of groundwater

While the pre-bomb atmospheric ${ }^{3} \mathrm{H}$ levels are not well known, water derived from precipitation before 1953 would have maximum ${ }^{3} \mathrm{H}$ concentrations of $\sim 0.1-0.4 \mathrm{TU}$ by 2003 (Kendall and Doctor 2011) and even less in 2012 (the sampling period). This implies that young groundwater in the study area should be identified by ${ }^{3} \mathrm{H}$ values $>1 \mathrm{TU}$. All ${ }^{3} \mathrm{H}$ values in the groundwater (2.4-3.1 $\mathrm{TU})$ were above the detection limit of $0.3 \mathrm{TU}$. Groundwater having ${ }^{3} \mathrm{H}$ concentrations greater than the detection limit is interpreted as water recharged after 1953
(Kendall and Doctor 2011). If the ${ }^{3} \mathrm{H}$ is greater than 0.5 $\mathrm{TU}$, it can be a strong evidence of the presence of some young (post-1952) water (Solomon and Cook 2000). Therefore, shallow groundwater in the Ndop plain was either recharged after 1952 or a considerable portion of the water was in equilibrium with the atmosphere since the early 1950s. This has also been reported in most shallow groundwater in Sub-Saharan Africa (Loehnert 1988; Njitchoua et al. 1997; Oga et al. 2008; Huneau et al. 2011; Fantong et al. 2010; Ako et al. 2012; Lapworth et al. 2013).

Knowledge of ${ }^{3} \mathrm{H}$ concentration in local precipitation, a prerequisite for groundwater apparent age interpretation 
Fig. 7 A spatial view of mean $\delta^{18} \mathrm{O}$ in groundwater of the study area (pink circle) relative to some studies in West Africa. They include southwest Cameroon (Ako et al. 2012); southwest Nigeria (Loehnert et al. 1988), Abeokuta, Nigeria (Lapworth et al. 2013); Voltaian Sedimentary Basin, Ghana (Acheampong and Hess 2000); Accra, Ghana (Kortatsi 2006); and Abidjan, Ivory Coast (Oga et al. 2008).

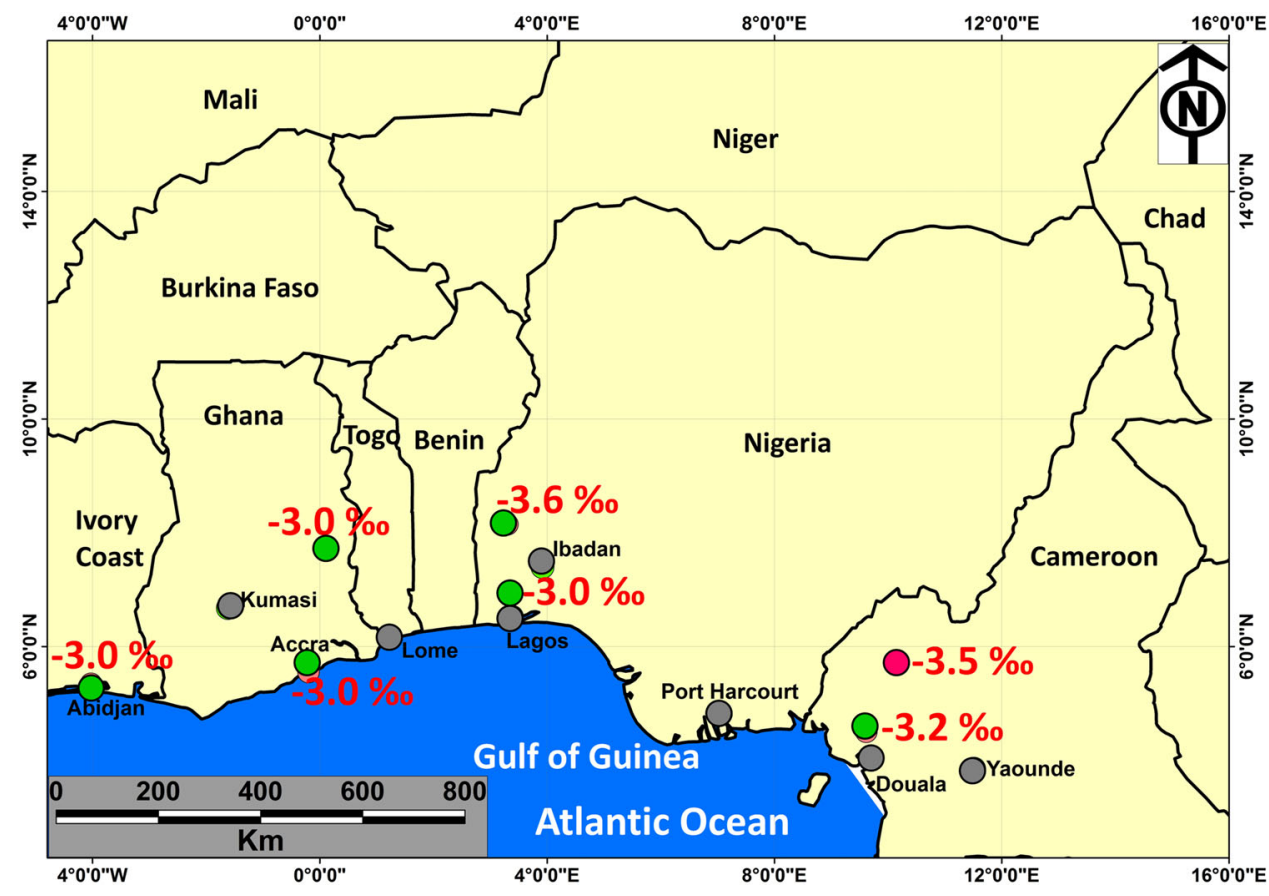

(Han et al. 2006; Lapworth et al. 2013) does not exist for the study area. However, ${ }^{3} \mathrm{H}$ records from 1960 to 2004 from IAEA stations in Douala (Cameroon), Yola (Nigeria) and Ndjamena (Chad), being the nearest to the study area were used in the apparent age determination (Fig. 8). Tritium decay curves in precipitation and groundwater (Fig. 8) were estimated from the decay equation:

$A=A_{0} \mathrm{e}^{-\lambda t}$

where $A_{0}$ is the initial ${ }^{3} \mathrm{H}$ activity, $A$ is the residual activity after decay over time $t$, and $\lambda$ is the decay constant which is

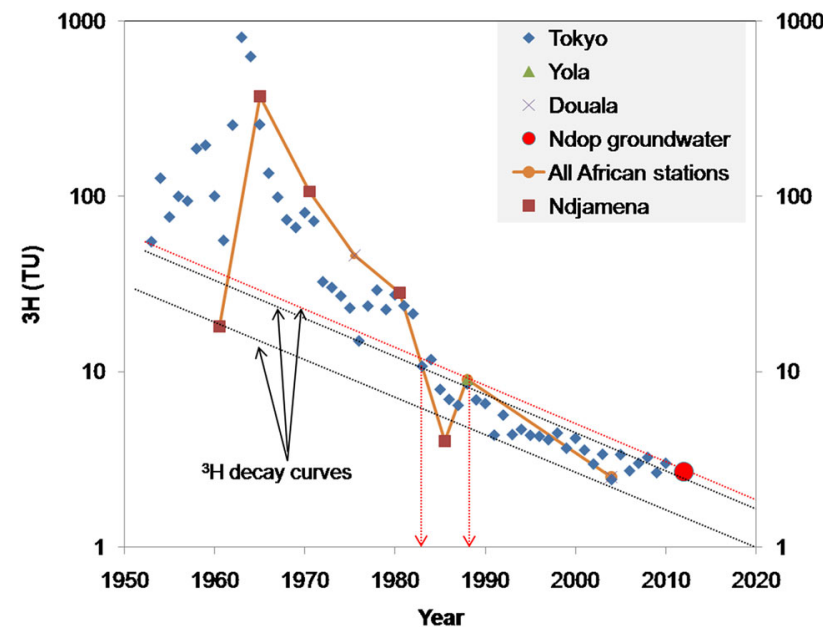

Fig. 8 Variation of ${ }^{3} \mathrm{H}$ in precipitation at Ndjamena (IAEA 2012), Yola (Onugba and Aboh 2009) and Douala (Ketchemen-Tandia et al. 2007; IAEA 2012) relative to Tokyo (IAEA 2012; http://www.iritokyo.jp/english/). The red circle represents the mean ${ }^{3} \mathrm{H}(2.7 \mathrm{TU})$ of groundwater in the Ndop Plain equal to 0.693 divided by the half-life of ${ }^{3} \mathrm{H}$ (12.32 years). From the intercepts of the average ${ }^{3} \mathrm{H}$ decay pattern in groundwater and precipitation pattern (Fig. 8), the qualitative recharge period was estimated between 1982 and 1988. Subsequently, the estimated residence time is $<30$ years, suggesting modern groundwater. Besides the low solubility of silicates (dominant in the study area), the observed low TDS values in groundwater corroborate the short residence time of the groundwater.

The mean ${ }^{3} \mathrm{H}$ in the groundwater is similar to that in 2004 rainfall $(2.5 \mathrm{TU})$ and groundwater in the coastal city of Douala (Ketchemen-Tandia et al. 2007) about $244 \mathrm{~km}$ from the study area. The average ${ }^{3} \mathrm{H}$ of precipitation from southwest Nigeria in 1979 (Loehnert 1988) and the Voltaian Sedimentary Basin of Ghana in 1994 (Acheampong and Hess 2000) are 12.3 and $3.4 \mathrm{TU}$, respectively. This confirms the observation that ${ }^{3} \mathrm{H}$ levels in young groundwater (as in this study) are usually about the same level as in modern rain (Acheampong and Hess 2000; Onugba and Aboh 2009). The low ${ }^{3} \mathrm{H}$ values are in agreement with its depleted atmospheric concentrations in the northern hemisphere especially at low latitudes (IAEA 2006). Despite the small sample size, the proposed recharge period and residence time constitute valuable information for water management in the Ndop plain.

Implications for future water management and climate variability

Given the vulnerability of surface water sources to the changing climate, shallow groundwater resources, which 
currently play an important role in providing sustainable drinking-water sources across Africa, will continue to do so in the future (Lapworth et al. 2013). The short residence time of the shallow groundwater as also reported (Loehnert 1988; Oga et al. 2008; Huneau et al. 2011; Fantong et al. 2010; Lapworth et al. 2013) and resilience of recharge to the changing climate (rainfall patterns) across Sub-Saharan Africa (MacDonald et al. 2009; Bonsor et al. 2011; Lapworth et al. 2013; Wirmvem et al. 2014a, b) in spite of different timing of recharge and rates still suggest good groundwater potentials across the region. The findings further provide evidence to support the affirmation that future changes in rainfall and recharge are unlikely to lead to failure of improved groundwater supplies in Africa (MacDonald et al. 2009; Bonsor et al. 2011; MacDonald et al. 2012; Lapworth et al. 2013).

\section{Conclusions}

The stable isotopes $\left(\delta^{18} \mathrm{O}\right.$ and $\left.\delta \mathrm{D}\right)$ and ${ }^{3} \mathrm{H}$ have enabled identification of shallow groundwater recharge process, timing and rate of recharge, and apparent recharge periodresidence time in the Ndop plain. Results suggest a single homogenous shallow unconfined aquifer that is being recharged by local rainfall through a direct diffuse/heterogeneous recharge mechanism. About $80 \%$ of the recharging rain infiltrates directly into the shallow aquifer system through minor openings in the unconsolidated sediments (under negligible evaporation effect); hence, vulnerable to any pollution at low elevation $(<1,260 \mathrm{~m})$ within the Ndop plain. Only $20 \%$ of the groundwater originates from localised recharge at high altitude in the surrounding mountainous chain or is mixed with inflowing streams and rivers from high elevations. The timing of recharge based on similarity in $\delta^{18} \mathrm{O}$ and $\delta \mathrm{D}$ in rain and groundwater is between the months of May and June characterized by abundant monsoon rains. Interestingly, recharge by the heaviest July-September monsoon rains is insignificant. The estimated recharge rate of $>251 \mathrm{~mm} /$ year (constituting at least $16 \%$ of annual rainfall) is high enough for the development of the groundwater resource for the prevailing agricultural practices in the area like rice farming. The qualitative recharge period (1983-1988) indicates the short residence time ( $<30$ years) of the groundwater. This suggests modern recharge, short circulation and recovery time (in case of contamination); hence, a renewable aquifer system. The data contribute a valuable tool for future water management in the Ndop plain. Future and long-term records of monthly stable isotopes in rainfall are recommended to shed more light on groundwater recharge investigations in northwest Cameroon and the entire Sub-Saharan Africa.
Acknowledgments The paper constitutes part of data generated during the $\mathrm{PhD}$ research of the corresponding author in Tokai University, Japan under the Japanese Government (MONBUKAGAKUSHO) Scholarship programme from the Ministry of Education, Culture, Sports, Science and Technology (MEXT). Material support was provided by Japan Science and Technology Agency (JST) and Japan International Cooperation Agency (JICA) under the Science and Technology Research Partnership for Sustainable Development (SATREPS) project titled: Magmatic Fluid Supply into Lakes Nyos and Monoun, and Mitigation of Natural Disasters in Cameroon. We thank the Dr Joseph Victor Hell and Dr Gregory Tanyileke of IRGM for their administrative assistance during this study. The manuscript was significantly improved by review comments from two anonymous reviewers.

Open Access This article is distributed under the terms of the Creative Commons Attribution License which permits any use, distribution, and reproduction in any medium, provided the original author(s) and the source are credited.

\section{References}

Acheampong SY, Hess JW (2000) Origin of the shallow groundwater system in the southern Voltaian sedimentary basin of Ghana: an isotopic approach. J Hydrol 233:37-53

Adanu EA (1991) Source and recharge of groundwater in the basement terrain in the Zaria-Kaduna area, Nigeria: applying stable isotopes. J Afr Earth Sci 13:229-234

Adelana SAM, MacDonald AM (2008) Groundwater research issues in Africa. In: Adelana SAM, MacDonald AM (eds) Applied groundwater studies in Africa. Taylor and Francis, London, pp 1-8

Ako AA, Shimada J, Hosono T, Ichiyanagi K, Nkeng GE, Eyong GET, Roger NN (2012) Hydrogeochemical and isotopic characteristics of groundwater in Mbanga, Njombe and Penja (Banana plain)-Cameroon. J Afr Earth Sci 75:25-36

Asaah ANE, Yokoyama T, Aka FT, Usui T, Wirmvem MJ, Chako Tchamabe B, Ohba T, Tanyileke G, Hell JV (2014) A comparative review of petrogenetic processes beneath the Cameroon Volcanic Line: geochemical constraints. Geosci Front. doi:10.1016/j.gsf.2014.04.012

Asomaning G (1992) Groundwater resources of the Birmin basin in Ghana. J Afr Earth Sci 15:375-384

Ayonghe SN (2001) A quantitative evaluation of global warming and precipitation in Cameroon from 1930 to 1995 and projections to 2060: effects on environment and water resources. In: Lambi $\mathrm{CM}$ (ed) Environmental issues: problems and prospects. Unique Printers, Bamenda, pp 142-155

Bonsor HC, MacDonald AM, Calow RC (2011) Potential impact of climate change on improved and unimproved water supplies in Africa. RSC Issues Environ Sci Technol 31:25-50

Brand WA, Geilmann H, Crosson ER, Rella CW (2009) Cavity ringdown spectroscopy versus high-temperature conversion isotope ratio mass spectrometry: a case study on $\delta^{2} \mathrm{H}$ and $\delta^{18} \mathrm{O}$ of pure water samples and alcohol/water mixtures. Rapid Commun Mass Spectrom 23:1879-1884

Clark ID, Fritz P (1997) Environmental isotopes in hydrogeology. Lewis, New York

Coplen TB, Herczeg AL, Barnes C (2000) Isotope engineering-Using stable isotopes of water molecule to solve practical problems. In: Cook P, Herczeg AL (eds) Environmental tracers in catchment hydrology. Kluwer Academic Publishers, Dordrecht, pp 79-110

Craig H (1961) Isotopic variations in meteoric waters. Science 133:1702-1703 
Dansgaard W (1964) Stable isotope in precipitation. Tellus 16:436-468

Dassi L (2011) Investigation by multivariate analysis of groundwater composition in a multilayer aquifer system from North Africa: a multi-tracer approach. Appl Geochem 26:1386-1398

Deshpande RD, Bhattacharya SK, Jani RA, Gupta SK (2003) Distribution of oxygen and hydrogen isotopes in shallow groundwaters from southern India: influence of a dual monsoon system. J Hydrol 271:226-239

Dincer T (1968) The use of oxygen-18 and deuterium concentrations in the water balance of lakes. Water Resour Res 4:1289-1305

EACC (2010) Economic adaptation to climate change: Synthesis report. World Bank, Washington

Fantong WY, Satake H, Aka FT, Ayonghe SN, Asai K, Mandal AK, Ako AA (2010) Hydrochemical and isotopic evidence of recharge, apparent age, and flow direction of groundwater in Mayo Tsanaga river basin, Cameroon: bearings on contamination. Environ Earth Sci 60:107-120

Favreau G, Cappelaere B, Massuel S, Leblanc M, Boucher M, Boulain N, Leduc C (2009) Land clearing, climate variability, and water resources increase in semiarid southwest Niger: A review. Water Resour Res 45, W00A16

Fonge BA, Egbe EA, Fongod AN, Tening AS, Achu RM, Yinda GS, Mvondo ZEA (2012) Effects of land use on macrophyte communities and water quality in the Ndop wetland plain, Cameroon. J Agric Soc Sci 12:41-49

Fontes JC (1980) Environmental isotopes in groundwater hydrology. In: Fritz P, Fontes JC (eds) Handbook of environmental isotope geochemistry, vol 1., The terrestrial environmentA. Elsevier, Amsterdam, pp 75-140

Gat JR (2010) Isotope hydrology: A study of the water cycle. Series on environmental science and management. Vol 6. Imperial College Press, London

Gonfiantini R, Frohlich K, Araguás-Araguás L, Rozanski K (1998) Isotopes in groundwater hydrology. In: Kendall C, McDonell JJ (eds) Isotope tracers in catchment hydrology. Elsevier, Amsterdam, pp 203-246

Goni IB (2006) Tracing stable isotope values from meteoric water to groundwater in the southwestern part of the Chad basin. Hydrogeol J 14:742-752

Han LF, Gröning M, Plummer LN, Solomon DK (2006) Comparison of the CFC technique with other techniques $\left({ }^{3} \mathrm{H},{ }^{3} \mathrm{H} /{ }^{3} \mathrm{He},{ }^{85} \mathrm{Kr}\right)$. IAEA, Use of chlorofluorocarbons in hydrology: A guide book. IAEA, Vienna, pp 191-198

Huneau F, Dakoure D, Celle-Jeaton H, Vitvar T, Ito M, Traore S, Compaore NF, Jirakova H, Le Coustumer P (2011) Flow pattern and residence time of groundwater within the south-eastern Taoudeni sedimentary basin (Burkina Faso, Mali). J Hydrol 408:423-439

IAEA (2006) Use of chlorofluorocarbons in hydrology: A guide book. International Atomic Energy Agency, STI/PUB/1238, Vienna

IAEA, 2012. Water Resources Programme. http://www.univie.ac.at/ cartography/project/wiser/index.php. Accessed 05 June 2012

Ibrahim M, Favreau G, Scanlon BR, Seidel JL, Le Coz M, Demarty J, Cappelaere B (2014) Long-term increase in diffuse groundwater recharge following expansion of rainfed cultivation in the Sahel, West Africa. Hydrol Geol 22:1293-1305

Kebede S, Travi Y (2012) Origin of the $\delta^{18} \mathrm{O}$ and $\delta^{2} \mathrm{H}$ composition of meteoric waters in Ethiopia. Quartern Int 257:4-12

Kendall C, Doctor DH (2011) Stable isotope applications in hydrologic studies. In: Holland, H. D., Turekian, K. K. (eds) Isotope geochemistry, $1^{\text {st }}$ edn. Academic Press London, pp181220

Ketchemen-Tandia B, Ntamak-Nida MJ, Boum-Nkot S, Wonkam C, Emvoutou H, Ebonji Seth C, Aranyossy JE (2007) First results of the isotopic study $\left({ }^{18} \mathrm{O},{ }^{2} \mathrm{H},{ }^{3} \mathrm{H}\right)$ of the Douala Quaternary aquifer (Cameroon). In: IAEA (ed) Advances in isotope hydrology and its role in Sustainable water resources management (IAEA-CN 151/37). IAEA, Vienna

Kortatsi BK (2006) Hydrochemical characterization of groundwater in the Accra plains of Ghana. Environ Geol 50:299-311

Lapworth DJ, MacDonald AM, Tijani MN, Darling WG, Gooddy DC, Bonsor HC, Araguás-Araguás LJ (2013) Residence times of shallow groundwater in West Africa: implications for hydrogeology and resilience to future changes in climate. Hydrogeol $\mathbf{J}$ 21:673-686

Loehnert EP (1988) Major chemical and isotope variations in surface and subsurface waters of West Africa. J Afr Earth Sci 7:579-588

Lutz A, Thomas JM, Panorska A (2011) Environmental controls on stable isotope precipitation values over Mali and Niger, West Africa. Environ Earth Sci 62:1749-1759

Ma J, He J, Qi S, Zhu G, Zhao W, Edmunds WM, Zhao Y (2013) Groundwater recharge and evolution in the Unhang basin, northwestern China. Appl Geochem 28:19-31

MacDonald AM, Calow RC, Macdonald DMJ, Darling WG, Dochartaigh O, Brighid E (2009) What impact will climate change have on rural groundwater supplies in Africa? Hydrol Sci J 54:690-703

MacDonald AM, Bonsor HC, Dochartaigh O, Brighid E, Taylor RG (2012) Quantitative maps of groundwater resources in Africa. Environ Res Lett 7:024009. doi:10.1088/1748-9326/7/2/024009

Mache JR, Signing P, Njoya A, Kunyukubundo F, Mbey JA, Njopwouo D, Fagel N (2013) Smectite clay from the Sabga deposit (Cameroon): mineralogical and physicochemical properties. Clay Miner 48:499-512

Marzoli A, Renne PR, Piccrilo EM, Francesca C, Bellieni G, Melfi A, Nyobe JB, N'ni J (1999) Silicic magmas from the continental Cameroon Volcanic Line (Oku, Bambouto and Ngaoundere): ${ }^{40} \mathrm{Ar}^{39} \mathrm{Ar}$ dates, petrology, $\mathrm{Sr}-\mathrm{Nd}-\mathrm{O}$ isotopes and their petrogenetic significance. Contrib Mineral Petr 135:133-150

Mbonu M, Travi Y (1994) Labelling of precipitation by stable isotopes $\left({ }^{18} \mathrm{O},{ }^{2} \mathrm{H}\right)$ over the Jos Plateau and the surrounding plains, north-central Nigeria. J Afr Earth Sci 19:91-98

Molua EL, Lambi CM (2006) Climate hydrology and water resources in Cameroon. CEEPA, Pretoria

Ndzeidze SK (2008) Detecting changes in a wetland using multispectral and temporal Landsat in the Upper Noun Valley drainage basin-Cameroon. MSc Thesis, Geography. Oregon State University

Neba A (1999) Modern geography of the Republic of Cameroon, 3rd edn. Neba Publishers, Bamenda

Negrel P, Pauwels H, Dewandel B, Gandolfi JM, Massacre C, Ahmed S (2011) Understanding groundwater systems and their functioning through the study of stable water isotopes in a hard-rock aquifer (Maheshwaram watershed, India). J Hydrol 397:55-70

Njitchoua R, Diver L, Fonts J-Ch, Naah E (1997) Geochemistry, origin and recharge mechanism of groundwaters from the Garoua sandstone aquifer, northern Cameroon. J Hydrol 190:123-140

Nkotagu H (1996) Application of environmental isotopes to groundwater recharge studies in a semi-arid fractured crystalline basement area of Dodoma, Tanzania. J Afr Earth Sci 22:443-457

Oga MS, Marlin C, Dever L, Filly A, Njitchoua R (2008) Hydrochemical and isotopic characteristics of coastal groundwater near Abidjan (southern Ivory Coast). In: Adelana SAM, MacDonald AM (eds) Applied groundwater studies in Africa. Taylor and Francis, London, pp 371-389

Onugba A, Aboh HO (2009) The tritium content of precipitation and groundwater at Yola, Nigeria. Sci World J 4:23-28

Owor M, Taylor RG, Tindimugaya C, Mwesigwa D (2009) Rainfall intensity and groundwater recharge: evidence from the Upper Nile Basin. Environ Res Lett 4:035009

Plummer LN, Böhkle JK, Busenberg E (2003) Approaches for groundwater dating. In: Lindsey BD, Phillips, SW, Donnelly CA,

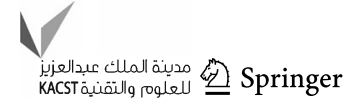


Speiran GK, Plummer LN, Böhlke JK, Focazio MJ, Burton WC, Busenberg E (eds) Residence times and nitrate transport in ground water discharging to streams in the Chesapeake bay watershed: U.S. Geological Survey Water-Resources Investigations Report 03-4035, pp 12-24

Solomon DK, Cook PG $(2000){ }^{3} \mathrm{H}$ and ${ }^{3} \mathrm{He}$. In: Cook PG, Herczeg AL (eds) Environmental tracers in catchment hydrology. Kluwer Academic Publishers, Dordrecht, pp 397-424

Takounjou AF, Ngoupayou JRN, Riotte J, Takem GE, Mafany G, Marechal JC, Ecokdeck GE (2011) Estimation of groundwater recharge of shallow aquifer on humid environment in Yaounde, Cameroon using hybrid water-fluctuation and hydrochemistry methods. Environ Earth Sci 64:107-118

Tanyileke GZ, Kusakabe M, Evans WC (1996) Chemical and isotopic characteristics of fluids along the Cameroon Volcanic Line, Cameroon. J Afr Earth Sci 22:433-441

Taupin J-D, Coudrain-Ribstein A, Gallaire R, Zuppi GM, Filly A (2000) Rainfall characteristics $\left(\delta^{18} \mathrm{O}, \delta^{2} \mathrm{H}, \Delta \mathrm{T}\right.$ and $\left.\Delta \mathrm{Hr}\right)$ in western Africa: regional scale and influence of irrigated areas. J Geophys Res 105:11911-11924

Taylor RG, Howard KWF (1996) Groundwater recharge in the Victoria Nile basin of East Africa: support for the soil moisture balance method using stable isotope and flow modelling studies. J Hydrol 180:31-53

Taylor RG, Scanlon B, Döll P et al (2013a) Ground water and climate change. Nat Clim Change 3:322-329

Taylor RG, Todd MC, Kongola L, Maurice L, Nahozya E, Sanga H, MacDonald AM (2013b) Evidence of the dependence of groundwater resources on extreme rainfall in East Africa. Nat Clim Change 3:374-378

Winter TC, Harvey JW, Franke OL, Alley WM (1998) Ground water and surface water a single resource. U.S. Geological Survey Circular 1139. http://pubs.usgs.gov/circ/circ1139/index.html\#pdf

Wirmvem MJ (2014) Hydrochemical and environmental tracer characterization of water resources in the Ndop Plain and Bamenda Highlands, North West Cameroon, Central Africa. $\mathrm{PhD}$ Thesis, Tokai University

Wirmvem MJ, Ohba T, Fantong WY, Ayonghe SN, Suila JY, Asaah ANE, Tanyileke G, Hell JV (2013) Hydrochemistry of shallow groundwater and surface water in the Ndop plain, North West Cameroon. Afr J Environ Sci Technol 7:518-530

Wirmvem MJ, Ohba T, Suila JY, Fantong WY, Bate NO, Seigo O, Wotany ER, Asaah ANE, Ayonghe SN, Tanyileke G, Hell JV (2014a) Hydrochemical and isotopic characteristics of groundwater in the Ndop plain, North West Cameroon: resilience to seasonal climatic changes. Environ Earth Sci 72:3585-3598

Wirmvem MJ, Ohba T, Fantong WY, Ayonghe SN, Suila JY, Asaah ANE, Asai K, Tanyileke G, Hell JV (2014b) Monthly $\delta^{18} \mathrm{O}, \delta \mathrm{D}$ and $\mathrm{Cl}^{-}$characteristics of precipitation in the Ndop plain, Northwest Cameroon: baseline data. Quat Int 338:35-41

Yidana SM, Chegbeleh LP (2013) The hydraulic conductivity field and groundwater flow in the unconfined aquifer system of the Keta Strip, Ghana. J Afr Earth Sci 86:45-56 